\title{
Fenômeno Fuller em problemas de controle ótimo: trajetórias em tempo mínimo de veículos autônomos subaquáticos
}

\author{
Eduardo Oda
}

\author{
DissertaÇÃo APRESENTADA \\ $\mathrm{AO}$ \\ Instituto De Matemática e Estatística \\ DA \\ Universidade de SÃo Paulo \\ PARA \\ OBTENÇÃO DO TÍTULO \\ $\mathrm{DE}$ \\ Mestre em CiênCias
}

\begin{abstract}
Área de Concentração: Matemática Aplicada
Orientador: Prof. Dr. Pedro Aladar Tonelli
\end{abstract}

Durante o desenvolvimento deste trabalho o autor recebeu auxílio financeiro do CNPq

São Paulo, 3 de julho de 2008 


\section{Fenômeno Fuller em problemas de controle ótimo: trajetórias em tempo mínimo de veículos autônomos subaquáticos}

Este exemplar corresponde à redação final da dissertação devidamente corrigida e defendida por Eduardo Oda e aprovada pela Comissão Julgadora.

Banca Examinadora:

- Prof. Dr. Pedro Aladar Tonelli (orientador) - IME-USP.

- Prof. Dr. Manuel Valentim de Pera Garcia - IME-USP.

- Prof. Dr. Felipe Miguel Pait - EP-USP. 
Aos meus pais, minhas irmãs, meus cunhados, meus sobrinhos e em especial, à minha Ana 


\section{Agradecimentos}

Registro aqui meus sinceros agradecimentos.

Ao professor Pedro A. Tonelli, por sua presença e paciência na minha orientação, o que possibilitou a realização deste trabalho.

Aos professores Manuel Valentim de Pera Garcia, Sônia Reina Leite Garcia

e Ângelo Barone Netto, que não medem esforços para ensinar, a mim e a todos os meus colegas e que, muito mais que professores, são nossos amigos.

À Ana, por sua contínua presença em minha vida, sempre me ajudando e me apoiando.

Aos meus pais, pela presença e apoio e por terem me dado condições de chegar até aqui.

Às minhas irmãs e demais familiares, por aturarem meu mau-humor e minha ausência nos últimos tempos.

A todos que me ajudaram na redação e revisão deste texto, em particular ao amigo Ricardo Freire, que teve a paciência de ler e corrigir cada pequeno detalhe e à amiga Marina Andretta, a quem incomodei inúmeras vezes com minhas dúvidas.

Aos amigos do IMEUSP, Alexandre Hannud Abdo, Fabio Niski, KBP, Ricardo Ribeiro e tantos outros, com os quais pude contar em cada momento de minha trajetória e com os quais, ao apoiar, aprendi muito. 
In the town where I was born...

Lennon/McCartney 


\section{Resumo}

As equações do modelo bidimensional de veículos autônomos subaquáticos fornecem um exemplo de sistema de controle não linear com o qual podemos ilustrar propriedades da teoria de controle ótimo. Apresentamos, sistematicamente, como os conceitos de formalismo hamiltoniano e teoria de Lie aparecem de forma natural neste contexto.

Para tanto, estudamos brevemente o Princípio do Máximo de Pontryagin e discutimos características de sistemas afins. Tratamos com cuidado do Fenômeno Fuller, fornecendo critérios para decidir quando ele está ou não presente em junções, utilizando para isso uma linguagem algébrica. Apresentamos uma abordagem numérica para tratar problemas de controle ótimo e finalizamos com a aplicação dos resultados ao modelo bidimensional de veículo autônomo subaquático.

Palavras-chave: teoria geométrica de controle, controle ótimo, Princípio do Máximo de Pontryagin, sistemas hamiltonianos, chattering, Fenômeno Fuller, controle singular, bang-bang. 


\section{Abstract}

The equations of the two-dimensional model for autonomous underwater vehicles provide an example of a nonlinear control system which illustrates properties of optimal control theory. We present, systematically, how the concepts of the Hamiltonian formalism and the Lie theory naturally appear in this context.

For this purpose, we briefly study the Pontryagin's Maximum Principle and discuss features of affine systems. We treat carefully the Fuller Phenomenon, providing criteria to detect its presence at junctions with an algebraic notation. We present a numerical approach to treat optimal control problems and we conclude with an application of the results in the bidimesional model of autonomous underwater vehicle.

Keywords: geometric control theory, optimal control,Pontryagin Maximum Principle, Hamiltonian systems, chattering, Fuller Phenomenon, singular control, bang-bang. 


\section{Sumário}

Definições $\quad$ vii

1 Introdução 1

1.1 Conceitos básicos . . . . . . . . . . . . . . . . . . . . 2

2 Controle ótimo $\quad 6$

2.1 O problema de controle ótimo . . . . . . . . . . . . . . . . . 7

2.2 O Princípio do Máximo de Pontryagin . . . . . . . . . . . . . . . . . . 10

2.3 Aplicando o Princípio do Máximo de Pontryagin . . . . . . . . . . . . . 12

2.4 O Princípio do Bang-bang . . . . . . . . . . . . . . . . 18

2.5 O Fenômeno Fuller . . . . . . . . . . . . . . . . . . . . . . 19

2.5.1 O exemplo de Fuller . . . . . . . . . . . . . . . . . 20

2.5.2 Um critério para verificar a presença do Fenômeno Fuller . . . . 26

2.6 Tratamento numérico de problemas de controle ótimo . . . . . . . . . . 41

2.6.1 Método direto . . . . . . . . . . . . . . . 41

2.6.2 Aproximação das variáveis adjuntas . . . . . . . . . . . . . 43

3 Submarino automático $\quad 46$

3.1 Trajetórias curiosamente não ótimas . . . . . . . . . . . . . . . . . . . 47

3.2 Simetrias do sistema . . . . . . . . . . . . . . . . . 51

3.3 Resultados numéricos . . . . . . . . . . . . . . . . . . . . 52

4 Conclusões $\quad 58$

A Listagem dos programas $\quad 59$

A.1 Programa para cálculo da ordem do problema . . . . . . . . . . . . 59

A.2 Scripts AMPL . . . . . . . . . . . . . . . . . 60 


\section{Definições}

Na lista abaixo $T$ é um espaço topológico, $M$ é uma variedade diferenciável e $x \in M$.

$[a, b] \quad$ Intervalo da reta tal que $a<b$. Se $b=+\infty$ então $[a, b]=[a,+\infty[$.

Mes $(T)$ Conjunto das funções mensuráveis $u:[a, b] \rightarrow T$.

$\mathrm{AC}(M)$ Conjunto das curvas absolutamente contínuas $x:[a, b] \rightarrow M$.

$\Sigma \quad$ Sistema de controle.

P Problema de controle ótimo.

$\operatorname{sign}(a)$ Sinal do número real $a$. Se $a=0$ então $\operatorname{sign}(a)$ não está definido.

$\dot{x}(t) \quad$ Derivada com relação a $t$ de uma função $x(t)$.

$x^{(k)}(t) \quad$ k-ésima derivada com relação a $t$ de uma função $x(t)$.

$T_{x} M \quad$ Espaço tangente a $M$ no ponto $x$.

TM Fibrado tangente de $M$.

$T_{x}^{*} M \quad$ Espaço cotangente a $M$ no ponto $x$, dual de $T_{x} M$.

$T^{*} M \quad$ Fibrado cotangente de $M$. 


\section{Capítulo 1}

\section{Introdução}

Um sistema de controle é um sistema cuja evolução pode ser influenciada por um agente externo: o controle. Por vezes isto é intencional. Por exemplo, é completamente indispensável que possamos controlar a velocidade e a direção de um veículo. Por vezes, simplesmente temos um sistema que não pode ser alterado e desejamos verificar até que ponto podemos influenciá-lo. Por exemplo, atividades metabólicas do nosso corpo.

Um ponto importante é que muitas vezes o estado não pode ser completamente conhecido. Então toda a informação que temos do sistema está limitada a uma função do estado: a saída.

Em linhas gerais, podemos pensar no controle como atuadores - acelerador, freio e na saída como os sensores - velocímetros, visão.

Do ponto de vista matemático, representamos esse tipo de sistema por um par de equações: uma equação diferencial ordinária da forma $\dot{x}(t)=f(x(t), u(t))$ e outra equação da forma $y(t)=h(x(t))$.

Neste caso, $x \in \mathbb{R}^{n}$ é a representação do estado do sistema que evolui com o tempo. A aplicação $f: \mathbb{R}^{n} \times \mathbb{R}^{m} \rightarrow \mathbb{R}^{n}$ nos conta como se dá essa evolução e $u \in \mathbb{R}^{m}$ é um parâmetro que influencia na evolução. O vetor $y \in \mathbb{R}^{p}$ é a informação disponível do sistema e $h: \mathbb{R}^{n} \rightarrow \mathbb{R}^{p}$ é a aplicação que mapeia o estado à informação que ele fornece. 
Nesta abordagem, um controle propriamente dito é uma aplicação $t \mapsto u(t)$ cuja imagem está no espaço dos parâmetros.

Lembre-se que, se escolhermos um controle mensurável e limitado e se $f$ for de classe $\mathcal{C}^{1}$ com respeito a primeira variável, então o teorema de existência da solução de Carathéodory garante a existência de uma solução absolutamente contínua para o problema de Cauchy $\dot{x}=f(x, u) ; x\left(t_{0}\right)=x_{0}$. Assim fica bem definido o par $(x, u)$ ao qual chamaremos de trajetória do sistema.

O nosso trabalho é encontrar um controle que faça o sistema se comportar da maneira desejada. E muita vezes esta maneira de se comportar é traduzida em dois subconjuntos do espaço de estados: o conjunto de partida e o conjunto de chegada.

Enfim, nosso problema fica traduzido na procura por controles, e portanto trajetórias, que atendam as especificações desejadas.

Este trabalho organiza-se em três partes. Na primeira parte apresentamos brevemente o formalismo básico utilizado para tratar estes problemas. Na segunda parte introduzimos os problemas de controle ótimo e discutimos alguns tópicos interessantes desta classe de problemas, em particular o Fenômeno Fuller. Por fim, na terceira parte aplicamos os resultados obtidos a um modelo idealizado de um veículo submarino automático.

\subsection{Conceitos básicos}

Esta sessão é dedicada à introdução de conceitos, definições e notações que serão utilizadas nos demais capítulos. Seguiremos a notação utilizada por Kupka em [1].

Definição 1.1.1 (Sistema não linear de controle). Um sistema não linear de controle é uma quintupla $\Sigma=(U, X, Y, f, h)$ onde:

(i) X e Y são variedades diferenciáveis;

(ii) U é um subespaço topológico de uma variedade $M U$; 
(iii) $f: X \times U \rightarrow T X$ é um campo vetorial em $X$ parametrizado em $U$ com uma extensão diferenciável em $X \times M U$;

(iv) $h: X \rightarrow Y$ é uma aplicação.

Definição 1.1.2 (Controle). Dado um sistema não linear de controle $\Sigma$, uma aplicação $u:[a, b] \rightarrow U, u \in \operatorname{Mes}(U)$ é chamada de controle.

Definição 1.1.3 (Controles admissíveis). Um subconjunto $\mathcal{U} \subset \operatorname{Mes}(U)$ é dito um conjunto de controles admissiveis se satisfaz:

(i) se $u:[a, b] \rightarrow U \in \mathcal{U}$ e $\left[a^{\prime}, b^{\prime}\right] \subset[a, b]$ então $\left.u\right|_{\left[a^{\prime}, b^{\prime}\right]} \in \mathcal{U}$;

(ii) se $u:[a, b] \rightarrow U \in \mathcal{U}$ e $c \in \mathbb{R}$, se definirmos $u_{c}:[a-c, b-c] \rightarrow U$ como $u_{c}(t)=u(t+c)$, então $u_{c} \in \mathcal{U}$;

(iii) se $u:[a, b] \rightarrow U \in \mathcal{U}$ e $v:[b, c] \rightarrow U \in \mathcal{U}$, se definirmos a concatenação

$$
u * v(t)= \begin{cases}u(t), & \text { se } t \in[a, b[ \\ v(t), & \text { se } t \in[b, c]\end{cases}
$$

então $u * v \in \mathcal{U}$.

Um sistema munido de um conjunto de controles admissiveis é denotado por $\Sigma=$ $(U, X, Y, f, h, \mathcal{U})$. Quando omitido, assumiremos $\mathcal{U}=\operatorname{Mes}(U)$.

Observação 1.1.4. Ao lidar com um sistema $\Sigma=(U, X, Y, f, h, \mathcal{U})$ vamos nos referir a $X$ como o espaço de estados, a $Y$ como o espaço das saídas e a $U$ como espaço de entradas ou parâmetros. Além disso, se a saída do sistema não importar omitimos $Y$ e $h$, denotando o sistema simplesmente por $\Sigma=(U, X, F, \mathcal{U})$.

Definição 1.1.5 (Trajetória). Uma trajetória de um sistema $\Sigma$ é um par $(x, u):[a, b] \rightarrow$ $X \times U$ tal que: 
(i) $x \in \mathrm{AC}(X)$;

(ii) $u \in \mathcal{U}$;

(iii) $\dot{x}(t)=f(x(t), u(t))$, para quase todo $t \in[a, b]$;

(iv) se o par ( $\tilde{x}, u):\left[a^{\prime}, b^{\prime}\right] \rightarrow X \times U$ é tal que $\left[a^{\prime}, b^{\prime}\right] \cap[a, b] \neq \emptyset$ e satisfaz (i), (ii), (iii) e se existe $t^{\prime} \in\left[a^{\prime}, b^{\prime}\right]$ tal que $\tilde{x}\left(t^{\prime}\right)=x\left(t^{\prime}\right)$ então $\tilde{x}(t)=x(t)$, para todo $t \in\left[a^{\prime}, b^{\prime}\right] \cap[a, b]$.

Definição 1.1.6. Dados um sistema $\Sigma=(U, X, f, \mathcal{U})$, dois subconjuntos, $A \subset X e$ $B \subset \mathbb{R} \times X$, e um tempo $T>0$ definimos o conjunto:

$$
\operatorname{Tr}(A, B, \mathcal{U})=\left\{\begin{array}{r}
(x, u):[0, T(u)] \rightarrow X \times U, \text { trajetória de } \Sigma \text { com } \\
\\
x(0) \in A,(T(u), x(T(u))) \in B
\end{array}\right\}
$$

e o conjunto:

$$
\operatorname{Tr}(A, B, \mathcal{U}, T)=\{(x, u):[0, T(u)] \rightarrow X \times U,(x, u) \in \operatorname{Tr}(A, B, \mathcal{U}), T(u)=T\}
$$

Definição 1.1.7 (Produto de Lie). Sejam dois campos vetoriais diferenciáveis, $f$ e $g$, definidos em $X$. Interpretando $f$ e $g$ como elementos da álgebra das derivações em $\mathcal{C}^{\infty}(X)$, podemos definir um novo campo vetorial, chamado de produto de Lie, como:

$$
[f, g]=f g-g f
$$

Ao longo deste trabalho sempre usaremos o produto de Lie em coordenadas:

$$
[f, g]=\frac{\partial g}{\partial x} f-\frac{\partial f}{\partial x} g
$$

Podemos calcular o produto de Lie de um campo vetorial $g$ repetidamente com um 
campo vetorial $f$, i.e., $[f,[f, \ldots[f, g] \ldots]]$. Para abreviar essa expressão, introduzimos a notação:

$$
[f,[f, \ldots[f, g] \ldots]]=a d_{f}^{k} g=\left[f, a d_{f}^{k-1}\right]
$$

$\operatorname{com} a d_{f}^{0} g=g$.

Neste trabalho vamos sempre considerar que, dado um sistema $\Sigma=(U, X, Y, f, h, \mathcal{U})$, a variedade $X$ não tem bordo, a aplicação $f$ é contínua e de classe $\mathcal{C}^{\infty}$ na primeira variável e a matriz Jacobiana de $f$ com respeito a $x$ é contínua com respeito às duas variáveis. 


\section{Capítulo 2}

\section{Controle ótimo}

Dado um sistema de controle e as especificações para seu funcionamento, obtemos um conjunto de trajetórias admissíveis. Podemos pensar em escolher, dentre estas trajetórias, uma que tenha menor custo segundo algum critério. Este é o problema de controle ótimo.

O custo de uma trajetória $(x, u):[a, b] \rightarrow X \times U$ pode ser descrito como $C(x, u)=$

$\int_{a}^{b} c(x(t), u(t)) d t$, onde $c: X \times U \rightarrow \mathbb{R}$ é chamada de função custo. Assim, o problema de controle ótimo traduz-se em procurar uma trajetória $(\bar{x}, \bar{u})$ que minimize $C$ e, quando tal trajetória existir, diremos que ela é uma trajetória ótima.

Poderíamos procurar por trajetórias ótimas utilizando o Cálculo das Variações Clássico. Mas neste caso, não saberíamos lidar com restrições mais sofisticadas nos controles admissíveis. Além disso, estaríamos limitados aos controles diferenciáveis.

Em 1961, Lev Semenovich Pontryagin publicou seu famoso livro The mathematical theory of optimal process [2], no qual discute este problema. Neste trabalho ele enuncia e prova o Princípio do Máximo de Pontryagin. Ainda hoje esta é a principal ferramenta para o projeto de controles ótimos explícitos.

O Princípio do Máximo de Pontryagin pode ser pensado como uma generaliazação das equações de Euler-Lagrange do Cálculo das Variações Clássico e, neste sentido, 
estabelece condições necessárias para uma trajetória ser ótima, restringindo nossa busca a um conjunto muito menor de trajetórias, conhecidas como extremais.

Note que nada se diz sobre condições suficientes. Portanto, ainda que encontremos uma trajetória extremal que satisfaça os pré-requisitos de partida e chegada, não podemos concluir que ela é uma trajetória ótima (mesmo que fosse a única a satisfazer as condições do Princípio do Máximo de Pontryagin e de contorno). Nessa hora contamos com o teorema de existência da solução do problema de controle ótimo, que pode nos ajudar a encerrar o assunto.

Ainda assim, nos problemas reais, sintetizar analiticamente um controle ótimo é uma tarefa árdua. De fato, envolve passos que geralmente não sabemos dar, como integrar sistemas hamiltonianos. Podemos, nestes casos, lançar mão de ferramentas numéricas, bastando encarar o problema de controle ótimo como um problema regular de programação não linear.

\subsection{O problema de controle ótimo}

Nesta seção vamos definir com rigor o problema de controle ótimo e em seguida exemplificá-lo.

Definição 2.1.1 (Função custo). Uma função custo para um sistema $\Sigma=(U, X, f)$ é uma função $c: X \times U \rightarrow \mathbb{R}$.

Neste trabalho consideraremos apenas funções custo que sejam contínuas, de classe $\mathcal{C}^{\infty}$ na primeira variável e cuja matriz Jacobiana com respeito a $x$ é contínua com respeito às duas variáveis. Desse modo poderemos mais à frente estender convenientemente o campo $f$ sem ferir as hipóteses feitas sobre ele na seção anterior.

Definição 2.1.2 (Custo de uma trajetória). Dados um sistema $\Sigma=(U, X, f)$ e uma 
função custo $c$, o custo de uma trajetória $(x, u):[a, b] \rightarrow X \times U$ é definido como:

$$
C(x, u)=\int_{a}^{b} c_{+}(x(t), u(t)) d t-\int_{a}^{b} c_{-}(x(t), u(t)) d t
$$

onde $c_{+}=\sup (c, 0), c_{-}=\sup (-c, 0)$ e as integrais podem divergir, convencionando:

$$
\left\{\begin{array}{l}
+\infty-\alpha=+\infty \\
\alpha-(+\infty)=-\infty \\
+\infty-(+\infty)=+\infty
\end{array}\right.
$$

Definição 2.1.3 (Problema de controle ótimo). Dados um sistema $\Sigma=(U, X, f, \mathcal{U})$, uma função custo $c$ e dois subconjuntos $A \subset X$ e $B \subset \mathbb{R} \times X$, encontrar uma trajetória $(\bar{x}, \bar{u}) \in \operatorname{Tr}(A, B, \mathcal{U})$, tal que

$$
C(\bar{x}, \bar{u})=\inf \{C(x, u):(x, u) \in \operatorname{Tr}(A, B, \mathcal{U})\}
$$

Denotamos tal problema por $P=(\Sigma, c, A, B)$.

Definição 2.1.4 (Problema de controle ótimo com tempo fixo T). Dados um sistema $\Sigma=(U, X, f, \mathcal{U})$, uma função custo $c$, um tempo $T>0$ e dois subconjuntos $A \subset X e$ $B \subset \mathbb{R} \times X$, encontrar uma trajetória $(\bar{x}, \bar{u}) \in \operatorname{Tr}(A, B, \mathcal{U}, T)$, tal que

$$
C(\bar{x}, \bar{u})=\inf \{C(x, u):(x, u) \in \operatorname{Tr}(A, B, \mathcal{U}, T)\} .
$$

Denotamos tal problema por $P=(\Sigma, c, A, B, T)$.

Definição 2.1.5 (Trajetória ótima). Para os problemas acima, se existir uma trajetória $(\bar{x}, \bar{u})$ ela é chamada de trajetória ótima.

Exemplo 2.1.6 (Carro sujeito à aceleração). Um carro de massa $m$ move-se em linha 
reta. Queremos ir da posição $a$ com velocidade $b$, conhecidas, à posição 0 com velocidade 0 em tempo mínimo. Para isso podemos controlar sua aceleração $u$, mas esta é limitada por uma constante positiva $k,|u| \leq k$.

Chamando a posição do carro de $x_{1}$ e sua velocidade de $x_{2}$, podemos descrever esse sistema com a equação de ordem dois $\ddot{x}_{1}=u / m$, que pode ser escrita como um sistema de ordem um:

$$
\begin{aligned}
& \dot{x}_{1}=x_{2} \\
& \dot{x}_{2}=\frac{u}{m} .
\end{aligned}
$$

Então o espaço de estados é $X=\mathbb{R}^{2}$, o espaço de parâmetros é $U=[-k, k]$, o campo é $f\left(x_{1}, x_{2}, u\right)=\left(x_{2}, \frac{u}{m}\right)$ e o problema de controle ótimo fica:

$$
\begin{aligned}
& \Sigma=\left([-k, k], \mathbb{R}^{2},\left(x_{2}, \frac{u}{m}\right), \operatorname{Mes}([-k, k])\right) \\
& P=(\Sigma, 1,\{(a, b)\},\{(0,0)\}) .
\end{aligned}
$$

Se ao invés de uma linha reta, o carro se movesse ao longo de uma curva orientada $\gamma$ conhecida, o problema teria uma formulação muito parecida, bastando pensar em $x_{1}$ como a abscissa curvilínea. Então, para evitar derrapagens, poderíamos ter outra restrição para a aceleração: $x_{2}^{2}<k_{2} R\left(x_{1}\right)$, onde $R\left(x_{1}\right)$ é o raio de curvatura de $\gamma$ no ponto $x_{1}$.

Encerramos esta seção com o teorema de existência de soluções para problemas de controle ótimo. Para uma demonstração desse resultado, veja a referência [3].

Teorema 2.1.7 (Existência de trajetórias ótimas). Seja $P=(\Sigma, c, A, B)$ um problema de controle ótimo com $\Sigma=(U, X, f$, Mes $(U))$. Se o sistema for controlável, $f$ for limitada, $U$ for compacto, existir $C>0$ tal que $C \leq c(x, u)$ para todo $x \in X$ e $u \in U$ e 
se o conjunto

$$
\{f(x, u), c(x, u): u \in U\}
$$

é convexo para todo $x \in X$, então $P$ tem solução.

\subsection{O Princípio do Máximo de Pontryagin}

Esta sessão dedica-se a enunciar o Princípio do Máximo de Pontryagin, bem como seu análogo, o Princípio do Mínimo de Pontryagin. Por fim, comentamos como fica o Princípio do Máximo de Pontryagin no caso particular do $\mathbb{R}^{n}$.

Teorema 2.2.1 (Princípio do Máximo de Pontryagin). Dado um problema de controle ótimo $P=(\Sigma, c, A, B), \operatorname{com} \Sigma=(U, X, f, \mathcal{U})$, defina a função diferenciável:

$$
\begin{aligned}
H_{\lambda}: T^{*} X \times U & \longrightarrow \mathbb{R} \\
(p, u) & \longmapsto\langle p, f(x, u)\rangle-\lambda c(x, u)
\end{aligned}
$$

onde $x$ é a projeção de $p$ em $X$ e $\lambda$ um parâmetro. Se existe trajetória ótima $(\bar{x}, \bar{u})$ : $[0, \bar{T}] \rightarrow X \times U$ então existem $\lambda \in\{0,1\}$ e um levantamento de $\bar{x}, \bar{p}:[0, \bar{T}] \rightarrow T^{*} X$, tais que $H_{\lambda}(\bar{p}(\bar{T}), \bar{u}(\bar{T}))=0$ e

$$
(A d j)\left\{\begin{array}{l}
\frac{d \bar{p}}{d t}(t)=X_{H_{\lambda}}(\bar{p}(t), \bar{u}(t)) \\
H_{\lambda}(\bar{p}(t), \bar{u}(t))=\sup \left\{H_{\lambda}(\bar{p}(t), v) \mid v \in U\right\}
\end{array}\right.
$$

para quase todo $t \in[0, \bar{T}]$, onde $X_{H_{\lambda}}$ é o campo hamiltoniano associado à função $H_{\lambda}(\cdot, u)$.

E $\bar{p}$ satisfaz:

(i) $\bar{p}(t) \neq 0_{\bar{x}(t)}$, q.t. $t \in[0, \bar{T}]$;

(ii) $\left\langle\bar{p}(0), T_{\bar{x}(0)} A\right\rangle=0$; 
(iii) $\exists \mu \in \mathbb{R}$ tal que $\left\langle(\mu, \bar{p}(\bar{T})), T_{q} B\right\rangle=0$, onde $q=(\bar{T}, \bar{x}(\bar{T}))$ e $(\mu, \bar{p}(\bar{T})) \in T_{q}^{*}(\mathbb{R} \times$ $X)=T_{\bar{T}}^{*} \mathbb{R} \times T_{\bar{x}(\bar{T})}^{*} X$.

Chamaremos p de variável adjunta e as equações (Adj) de equações adjuntas.

Observação 2.2.2. As condições (ii) e (iii) são chamadas de condições de transversalidade. No caso em que $B$ é um ponto, assumimos que $T_{q} B=0$, e nesse caso (iii) não acrescenta informação. Uma observação análoga se aplica a (ii).

Definição 2.2.3 (Extremais). Toda curva $(p, u):[0, T] \rightarrow T^{*} X \times U$ tal que $p$ é absolutamente contínua, u é mensurável e satisfaz (Adj) para quase todo $t \in[0, T]$ é chamada extremal. Se $\lambda=1$ o extremal é dito ordinário e se $\lambda=0$ o extremal é dito excepcional.

Corolário 2.2.4. $H_{\lambda}$ é constante ao longo de extremais.

Observação 2.2.5 (Princípio do Mínimo de Pontryagin). Existe um análogo ao Princípio do Máximo de Pontryagin: o Princípio do Mínimo de Pontryagin. Seus enunciados são idênticos, com a diferença de que no Princípio do Mínimo de Pontryagin a hamiltoniana se define como $H_{\lambda}(p, u)=\langle p, f(x, u)\rangle+\lambda c(x, u)$ e as trajetórias ótimas satisfazem: $H_{\lambda}(\bar{p}(t), \bar{u}(t))=\inf \left\{H_{\lambda}(\bar{p}(t), v): v \in U\right\}$.

Para demostrar o Princípio do Mínimo de Pontryagin a partir do Princípio do Máximo de Pontryagin basta aplicar o Princípio do Máximo de Pontryagin e tomar $-p$ como as variáveis adjuntas do Princípio do Mínimo de Pontryagin.

Observação 2.2.6 (Problema de controle ótimo com tempo fixo T). O Princípio do Máximo de Pontryagin ainda é válido quando o tempo final está fixado, porém as condições de transversalidade podem não mais serem satisfeitas.

Exemplo 2.2.7 (Um caso especial do Princípio do Máximo de Pontryagin). Se $X$ é um aberto do $\mathbb{R}^{n}$ e $U$ é um aberto do $\mathbb{R}^{m}$ então um elemento do fibrado cotangente, 
$T^{*} X=X \times\left(\mathbb{R}^{n}\right)^{*}$, é um $\operatorname{par}(x, p), \operatorname{com} x=\left(x_{1}, \ldots, x_{n}\right) \in \mathbb{R}^{n}$ e $p=\left(p_{1}, \ldots, p_{n}\right) \in\left(\mathbb{R}^{n}\right)^{*}$ e as equações adjuntas ficam:

$$
(\operatorname{Adj})\left\{\begin{array}{l}
\frac{d \bar{x}_{i}}{d t}(t)=\frac{\partial H_{\lambda}}{\partial p_{i}}(\bar{x}(t), \bar{p}(t), \bar{u}(t)) \\
\frac{d \bar{p}_{i}}{d t}(t)=-\frac{\partial H_{\lambda}}{\partial x_{i}}(\bar{x}(t), \bar{p}(t), \bar{u}(t)) \\
H_{\lambda}(\bar{x}(t), \bar{p}(t), \bar{u}(t))=\sup \left\{H_{\lambda}(\bar{x}(t), \bar{p}(t), v) \mid v \in U\right\}
\end{array}\right.
$$

para $i=1, \ldots, n$, q.t. $t \in[0, \bar{T}]$, com $\bar{p}$ não identicamente nulo.

\subsection{Aplicando o Princípio do Máximo de Pontryagin}

O Princípio do Máximo de Pontryagin nos permite identificar as trajetórias extremais, a partir das quais podemos sintetizar uma trajetória ótima. Por sintetizar entendemos o ato de concatenar órbitas de extremais de modo a ir do conjunto de saída ao conjunto de destino.

Este passo é um dos mais complicados na determinação analítica do controle ótimo pois, mesmo quando conseguimos integrar o sistema hamiltoniano, nem sempre conseguimos facilmente encontrar a síntese que seja ótima.

Nesta seção veremos um exemplo clássico no qual a trajetória ótima é facilmente sintetizada e ilustramos todos os passos da metodologia de aplicação do Princípio do Máximo de Pontryagin.

Exemplo 2.3.1 (Carro sujeito à aceleração). Vamos aplicar o Princípio do Máximo de Pontryagin ao Exemplo 2.1.6. Para simplificar, supomos que $m=1$ e $k=1$.

O problema de controle ótimo é $P=(\Sigma, 1,\{(a, b)\},\{(0,0)\})$, onde o sistema é dado por $\Sigma=\left([-1,1], \mathbb{R}^{2},\left(x_{2}, u\right), \operatorname{Mes}([-1,1])\right)$. Então, definindo $H_{\lambda}(x, p, u)=p_{1} x_{2}+p_{2} u-$ 
$\lambda$, temos as equações adjuntas:

$$
\begin{cases}\frac{d \bar{x}_{1}}{d t}(t)=\bar{x}_{2}(t) & \frac{d \bar{p}_{1}}{d t}(t)=0 \\ \frac{d \bar{x}_{2}}{d t}(t)=\bar{u}(t) & \frac{d \bar{p}_{2}}{d t}(t)=-\bar{p}_{1}(t) \\ \bar{p}_{1}(t) \bar{x}_{2}(t)+\bar{p}_{2}(t) \bar{u}(t)-\lambda= & \sup \left\{\bar{p}_{1}(t) \bar{x}_{2}(t)+\bar{p}_{2}(t) v-\lambda \mid v \in\left[-k_{1}, k_{1}\right]\right\}\end{cases}
$$

Note que $\lambda$ não importa e então todos os extremais são ordinários e excepcionais.

Neste problema em particular, podemos encontrar facilmente formas gerais para $p_{1}$ e $p_{2}$, pois suas derivadas não dependem de $x$ :

$$
\begin{aligned}
& p_{1}=k_{1} \\
& p_{2}=-t k_{1}+k_{2} .
\end{aligned}
$$

O Princípio do Máximo de Pontryagin dispõe que a trajetória ótima deve ser tal que:

$$
H_{\lambda}(\bar{x}(t), \bar{p}(t), \bar{u}(t))=\sup \left\{H_{\lambda}(\bar{x}(t), \bar{p}(t), v) \mid v \in[-1,1]\right\}
$$

então, precisamos maximizar $H_{\lambda}$ com relação a $u$. Mas note que $H_{\lambda}$ é linear na variável $u$, então, num instante de tempo $t$, o máximo de $H_{\lambda}$ é atingido num dos extremos de $[-1,1]$

É fácil notar que a escolha do extremo é dada pelo sinal de $p_{2}(t)$. De fato, $p_{2}(t)$ é a inclinação da reta $H_{\lambda}(\bar{x}(t), \bar{p}(t), u)=u$. Isso fica claro na Figura 2.1, em que esta reta é desenhada para um tempo $t$ no qual $p_{2}(t)>0$. Note que quando $p_{2}(t)=0$, nada podemos afirmar sobre $u(t)$. 


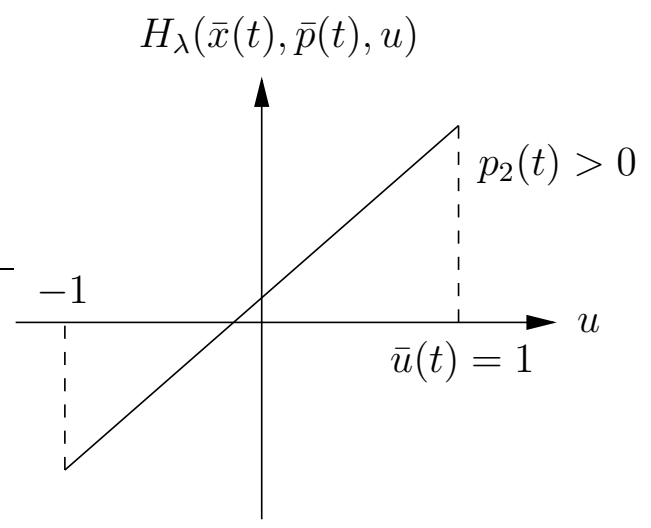

Figura 2.1: Reta $H_{\lambda}(\bar{x}(t), \bar{p}(t), u)=u \operatorname{com} p_{2}(t)>0$

Concluímos, assim, que o controle ótimo é:

$$
\bar{u}(t)= \begin{cases}1, & \text { se } \bar{p}_{2}(t)>0 \\ -1, & \text { se } \bar{p}_{2}(t)<0\end{cases}
$$

e nos pontos em que $\bar{p}_{2}(t)=0, \bar{u}(t)$ não está definido. Resumidamente escrevemos $u(t)=\operatorname{sign}\left(p_{2}(t)\right)$.

Apesar de aparentemente resolvido o problema, ainda não sabemos exatamente quem é $\bar{p}_{2}$, pois desconhecemos as constantes $k_{1}$ e $k_{2}$. A partir de agora vamos atacar o problema da síntese da trajetória ótima, comentado no início da seção.

Note que $\bar{p}_{2}$ é um polinônio de grau 1, trocando de sinal no máximo uma vez, logo $\bar{u}$ tem no máximo uma descontinuidade. Podemos então encontrar $\bar{x}_{1}$ e $\bar{x}_{2}$ em cada um dos intervalos onde $\bar{u}$ é constante. De fato, no intervalo em que $\bar{u} \equiv 1$, temos que:

$$
\begin{aligned}
& \bar{x}_{1}(t)=\frac{t^{2}}{2}+k_{3} t+k_{4} \\
& \bar{x}_{2}(t)=t+k_{3}
\end{aligned}
$$


e no intervalo em que $\bar{u} \equiv-1$, temos que:

$$
\begin{aligned}
& \bar{x}_{1}(t)=-\frac{t^{2}}{2}+k_{3} t+k_{4} \\
& \bar{x}_{2}(t)=-t+k_{3} .
\end{aligned}
$$

Na Figura 2.2 podemos observar os retratos de fase de cada uma destas soluções.

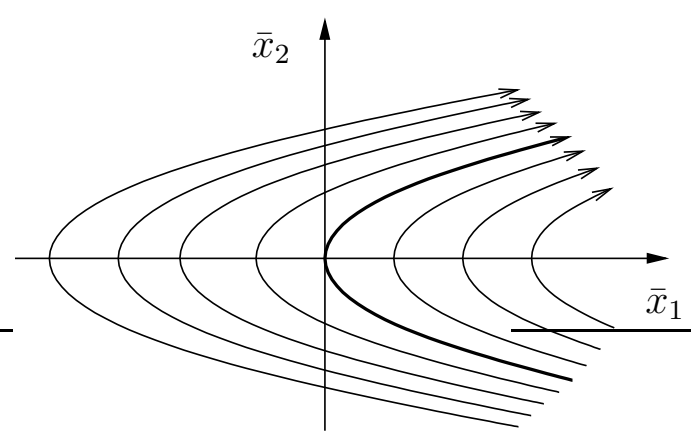

(a) $\bar{u} \equiv 1$

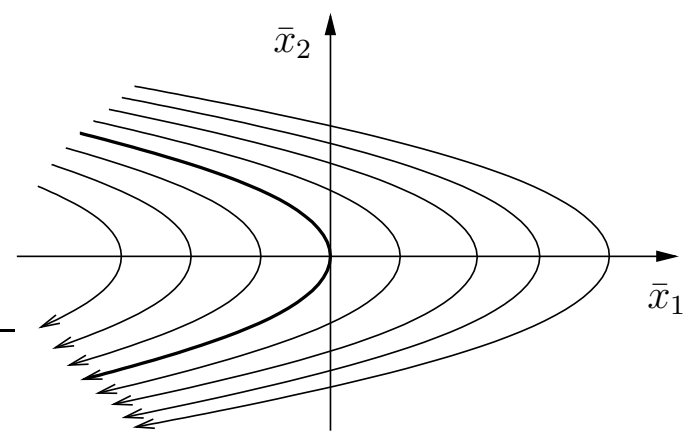

(b) $\bar{u} \equiv-1$

Figura 2.2: Retratos de fase

Claramente os extremais deste problema são concatenações de no máximo duas destas curvas, pois cada concatenação corresponde a uma descontinuidade no controle.

Lembre-se que o objetivo é encontrar um controle que faça o carro ir para o estado $(0,0)$. Vamos chamar de $S$ o passado da origem por cada uma das duas únicas órbitas que passam por ela (em destaque na Figura 2.2).

Fica claro que as únicas concatenações que podemos considerar são aquelas cujo último trecho está contido em $S$. Veja Figura 2.3.

Então, dado qualquer ponto $(a, b)$ do plano, precisamos dele chegar a $S$ sem trocar o sinal de $\bar{p}_{2}$, o que corresponderia a uma descontinuidade no controle. Evidentemente, no caso de $(a, b) \in S$ não será necessária nenhuma descontinuidade no controle.

Para fazer isso descobrimos uma expressão para $S$ usando condições iniciais nulas 


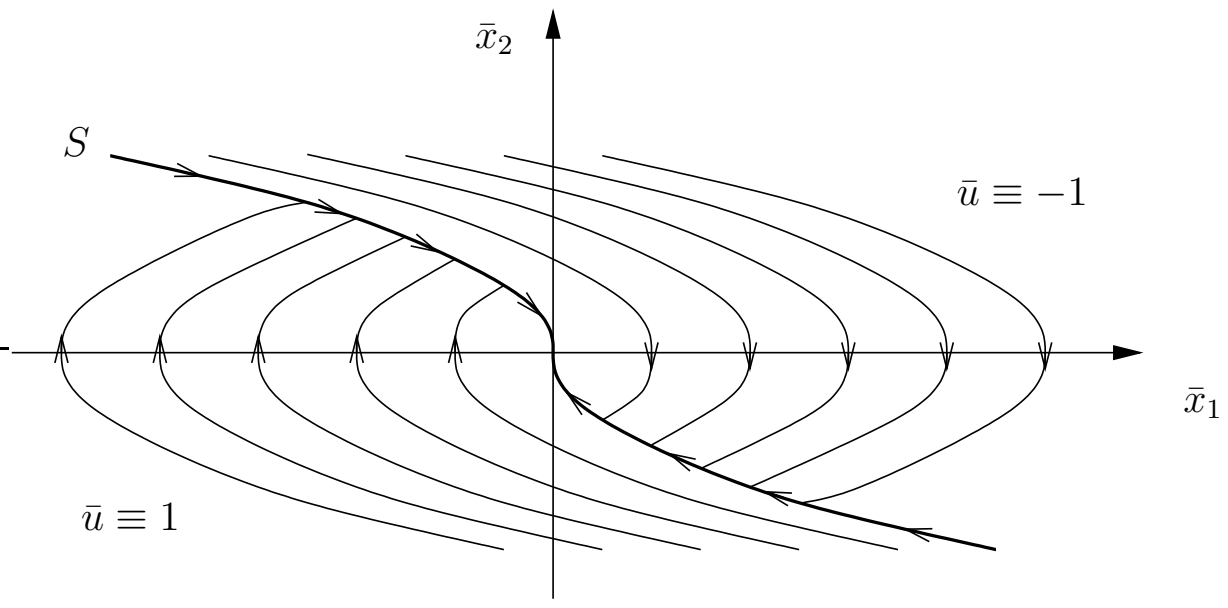

Figura 2.3: Trajetórias ótimas projetadas em $\mathbb{R}^{2}$

nas equações de $\dot{x}_{1}$ e $\dot{x}_{2}$ :

$$
S=\left\{\left(x_{1}, x_{2}\right) \in \mathbb{R}^{2} \mid x_{2} \leq 0 \text { e } 2 x_{1}=x_{2}^{2}\right\} \bigcup\left\{\left(x_{1}, x_{2}\right) \in \mathbb{R}^{2} \mid x_{2} \geq 0 \text { e } 2 x_{1}=-x_{2}^{2}\right\}
$$

Sabemos que por cada ponto do plano passam duas órbitas (uma para $\bar{u} \equiv 1$ e outra para $\bar{u} \equiv-1)$. É fácil verificar que apenas uma delas intercepta $S$ no futuro, momento no qual o controle muda.

Assim, para acabar de caracterizar o controle, basta encontrar o instante $t_{1}$, em que o carro chega em $S$, e o instante $t_{f}$, em que o carro chega na origem.

Por exemplo, suponha que $(a, b)$ está abaixo de $S$, i.e., $(a, b) \in S_{-}$, onde

$$
S_{-}=\left\{\left(x_{1}, x_{2}\right) \in \mathbb{R}^{2} \mid x_{2} \leq 0 \text { e } 2 x_{1}<x_{2}^{2}\right\} \bigcup\left\{\left(x_{1}, x_{2}\right) \in \mathbb{R}^{2} \mid x_{2} \geq 0 \text { e } 2 x_{1}<-x_{2}^{2}\right\}
$$

Neste caso devemos começar com $\bar{u}(t)=1,0 \leq t<t_{1}$. Para encontrar $t_{1}$ basta resolver $2 \bar{x}_{1}\left(t_{1}\right)=-\bar{x}_{2}^{2}\left(t_{1}\right)$, obtendo $t_{1}=-b+\frac{\sqrt{2 b^{2}-4 a}}{2}$.

No intervalo $t_{1}<t<t_{f}, \bar{u}(t)=-1$. Então, resolvendo $2 \bar{x}_{2}\left(t_{f}\right)=0$, onde $\bar{x}_{2}$ é a solução de $\dot{x}_{2}=1$ com a condição inicial $x_{2}\left(t_{1}\right)=\bar{x}_{2}\left(t_{1}\right)$, encontramos $t_{f}=-b+$ 
$\sqrt{2 b^{2}-4 a}$.

E com isso caracterizamos completamente o controle ótimo.

Examinando com cuidado o exemplo anterior vemos que alguns passos foram cruciais para encontrar a trajetória ótima:

(i) o Princípio do Máximo de Pontryagin garantiu que o controle assumia valores nos vértices de $U$;

(ii) pudemos facilmente encontrar uma expressão de $\bar{p}$, pois as equações diferenciais que $\bar{p}$ devia satisfazer não dependiam de $x$. De onde concluímos que o controle ótimo $\bar{u}$ era descontínuo em, no máximo, um ponto;

(iii) verificamos que, dado um ponto $(a, b)$, existia somente um controle que satisfazia as condições necessárias que o Princípio do Máximo de Pontryagin estabelece;

(iv) o problema satisfazia as condições necessárias para a existência de soluções.

Isto nos indica um procedimento de 4 passos que possivelmente encontra a solução do problema de controle ótimo:

Passo 1: usar o Princípio do Máximo de Pontryagin para descobrir propriedades das trajetórias ótimas;

Passo 2: usar as equações adjuntas do Princípio do Máximo de Pontryagin para caracterizar os extremais;

Passo 3: a partir dos extremais, sintetizar trajetórias que realizem as condições iniciais e finais e que tenham as propriedades descobertas no primeiro passo;

Passo 4: determinar se uma destas trajetórias é de fato uma trajetória ótima. 
Como comentado, o passo 2 pode ser muito complicado, pois depende da possibilidade de integrar um sistema hamiltoniano, qua sabemos só ser possível em alguns casos muito especiais.

Os passos 1 e 3 escondem alguns pontos importantes e, ao estudá-los com um pouco mais de cuidado, verificamos dois fenômenos interessantes: Princípio do Bang-bang e o Fenômeno Fuller, os quais discutimos nas duas sessões seguintes.

\subsection{O Princípio do Bang-bang}

No primeiro passo do procedimento proposto na sessão anterior, o Princípio do Máximo de Pontryagin nos permitiu descobrir uma importante propriedade do controle ótimo: ele saltava de um vértice para outro do espaço dos parâmetros de acordo com o sinal de uma função.

É fácil notar que esse fenômeno ocorre sempre que a hamiltoniana $H_{\lambda}$ for afim em $u$. Este comportamento, por causa dos saltos do controle ótimo, é conhecido por Princípio do Bang-bang.

Esta pode parecer uma situação bem particular, mas uma das classes de sistemas de controle mais importantes, por ser muito comum nos problemas aplicados, é a dos sistemas afins:

$$
\dot{x}=f(x)+\sum_{i=1}^{m} g_{i}(x) u_{i} .
$$

Se lidamos com um problema com esse tipo de dinâmica e se o custo também for afim em $u$ :

$$
c(x, u)=f^{0}(x)+\sum_{i=1}^{m} g_{i}^{0}(x) u_{i},
$$

a hamiltoniana fica afim em $u$ :

$$
H_{\lambda}(x, p, u)=\langle p, f\rangle-\lambda f^{0}+\sum_{i=1}^{m}\left(\left\langle p, g_{i}\right\rangle-\lambda g_{i}^{0}\right) u_{i} .
$$


Para simplificar, suponha que $m=1$, i.e., um sistema com uma entrada, e que $|u| \leq K$. Vamos denotar por $\psi=\langle p, f\rangle-\lambda f^{0}$ e por $\phi=\langle p, g\rangle-\lambda g^{0}$.

Mesmo neste caso particular podem aparecer muitas complicações. Mas se em um determinado intervalo $\phi \neq 0$, exceto em um subconjunto de medida nula, fica claro do Princípio do Máximo de Pontryagin que $u=K \operatorname{sign}(\phi)$ neste intervalo.

Um intervalo com essas características é chamado de intervalo não singular. A imagem deste intervalo pelo controle é chamado de arco não singular.

Analogamente, um intervalo em que $\phi \equiv 0$, é chamado de intervalo singular e a sua imagem pelo controle, de arco singular.

Uma junção é um instante $t$ para o qual existe $\epsilon$ tal que o intervalo $(t, t+\epsilon)$ - ou $(t+\epsilon, t)$, se $\epsilon<0$ - é singular e o intervalo $(t-\epsilon, t)-$ ou $(t, t-\epsilon)$, se $\epsilon<0$ - é não singular.

Para simplificar as discussões vamos livremente chamar um intervalo (não) singular de arco (não) singular, sem prejudicar o rigor.

\subsection{O Fenômeno Fuller}

Uma leitura crítica do procedimento proposto na Seção 2.3 logo revela que podemos ter muitas dificuldades no passo 3. Por exemplo, pode não ser possível sintetizar uma trajetória com um número finito de concatenações. O primeiro e mais famoso exemplo em que isso ocorre foi apresentado por A. T. Fuller em 1963.

Neste exemplo as descontinuidades do controle se acumulam num ponto. Tal característica levou esse fenômeno a ser conhecido por Chattering.

Mais tarde, em 1990, Kupka mostrou a "onipresença" do Chattering [4], que desde então passou a ser conhecido também como Fenômeno Fuller.

Surpreendentemente, o exemplo que Fuller apresentou é o mesmo sistema do carro

que tratamos nas últimas sessões, mas com uma função custo diferente: $c(t)=\frac{x_{1}^{2}}{2}$ e o 
tempo final fixo. Vejamos com detalhes esse exemplo.

\subsubsection{O exemplo de Fuller}

O problema de controle ótimo com tempo fixo é $P=\left(\Sigma, \frac{x_{1}^{2}}{2},\{(-3,2)\},\{(0,0)\}, 5\right)$, $\operatorname{com} \Sigma=\left([-1,1], \mathbb{R}^{2},\left(x_{2}, u\right), \operatorname{Mes}([-1,1])\right)$.

A estratégia para resolvê-lo segue os seguintes passos:

(i) verificar que existe uma trajetória ótima;

(ii) mostrar que a trajetória ótima é única;

(iii) mostrar que $\lambda=1$;

(iv) mostrar que acontece bang-bang;

(v) mostrar que o controle ótimo tem infinitas descontinuidades;

(vi) contruir uma trajetória que satisfaz as condições necessárias que o Princípio do Máximo de Pontryagin estabelece, supondo que as descontinuidades do controle ótimo ocorrem numa sequência de pontos em progressão geométrica.

O primeiro passo é simplesmente uma verificação do Teorema 2.1.7.

Para provar a unicidade, suponha que $(x, u)$ é uma trajetória ótima e considere uma outra trajetória $(y, v)$. Por hipótese, $C(x, u) \leq C(y, v)$. Vamos mostrar que $C(x, u)<C(y, v)$.

Podemos construir uma nova trajetória $(z, w)$ tal que $z=\rho x+(1-\rho) y$, com $\rho \in(0,1)$. E assim:

$$
\begin{aligned}
c(z, w) & =\frac{z_{1}^{2}}{2} \\
& =\frac{\left(\rho x_{1}+(1-\rho) y_{1}\right)^{2}}{2} \\
& =\rho c(x, u)+(1-\rho) c(y, v)-\frac{\rho(1-\rho)}{2}\left(x_{1}-y_{1}\right)^{2} .
\end{aligned}
$$


Então temos que:

$$
C(z, w)=\rho C(x, u)+(1-\rho) C(y, v)-\frac{\rho(1-\rho)}{2} \int_{0}^{T}\left(x_{1}-y_{1}\right)^{2} d t
$$

Por hipótese sabemos que $C(x, u) \leq C(z, w)$, assim

$$
C(y, v) \geq C(x, u)+\frac{\rho}{2} \int_{0}^{T}\left(x_{1}-y_{1}\right)^{2} d t>C(x, u)
$$

de onde concluímos que a trajetória ótima é única.

Definindo a hamiltoniana $H_{\lambda}(x, p, u)=p_{1} x_{2}+p_{2} u-\lambda \frac{x_{1}^{2}}{2}$, temos as equações adjuntas:

$$
\begin{cases}\frac{d \bar{x}_{1}}{d t}(t)=\bar{x}_{2}(t) & \frac{d \bar{p}_{1}}{d t}(t)=\lambda x_{1} \\ \frac{d \bar{x}_{2}}{d t}(t)=\bar{u}(t) & \frac{d \bar{p}_{2}}{d t}(t)=-\bar{p}_{1}(t) \\ \bar{p}_{1}(t) \bar{x}_{2}(t)+\bar{p}_{2}(t) \bar{u}(t)-\lambda \frac{\bar{x}_{1}^{2}}{2} & =\sup \left\{\bar{p}_{1}(t) \bar{x}_{2}(t)+\bar{p}_{2}(t) v-\lambda \frac{\bar{x}_{1}^{2}}{2} \mid v \in\left[-k_{1}, k_{1}\right]\right\} .\end{cases}
$$

Se $\lambda=0$, então $\bar{p}_{2}$ é um polinômio de grau 1. Assim, o controle $\bar{u}$ teria apenas uma descontinuidade. Com base nos resultados do Exemplo 2.3.1, vemos que neste caso não é possível satizfazer $T=5$, pois o carro chegaria no estado desejado em $t=-2+2 \sqrt{5}<5$ e seríamos forçados a fazer $\bar{u}(t)=0$ quando $t \in[-2+2 \sqrt{5}, 5]$, o que faria o controle ter duas descontinuidades. Portanto $\lambda=1$.

Como $H_{\lambda}$ é linear em $u$, vale o Princípio do Bang-bang, logo $\bar{u}$ vai assumir valores em $\{-1,1\}$, mais precisamente, $\bar{u}=\operatorname{sign}\left(\bar{p}_{2}\right)$. E, assim, para cada intervalo em que $\bar{u}$ 
for constante temos:

$$
\begin{aligned}
& \bar{x}_{1}= \pm \frac{t^{2}}{2}+t k_{1}+k_{2} \\
& \bar{x}_{2}= \pm t+k_{1} \\
& \bar{p}_{1}= \pm \frac{t^{3}}{6}+\frac{t^{2}}{2} k_{1}+t k_{2}+k_{3} \\
& \bar{p}_{2}=-\left( \pm \frac{t^{4}}{24}+\frac{t^{3}}{6} k_{1}+\frac{t^{2}}{2} k_{2}+t k_{3}\right)+k_{4} .
\end{aligned}
$$

Note que, não importa qual estratégia de controle, não podemos ir de $(-3,2)$ para a origem em $t<-2+2 \sqrt{5}$. Portanto, o problema não teria solução se exigíssemos que o tempo final fosse, por exemplo, 2. Por outro lado, se quiséssemos um tempo maior que $-2+2 \sqrt{5}$, bastaria chegar até a origem e ficar parado. Por exemplo no nosso caso, poderíamos fazer:

$$
u(t)= \begin{cases}1, & \text { se } 0 \leq t<-2+\sqrt{5} \\ -1, & \text { se }-2+\sqrt{5} \leq t<-2+2 \sqrt{5} \\ 0, & \text { se }-2+2 \sqrt{5}<t<5 .\end{cases}
$$

E o carro se comportaria como na Figura 2.4.
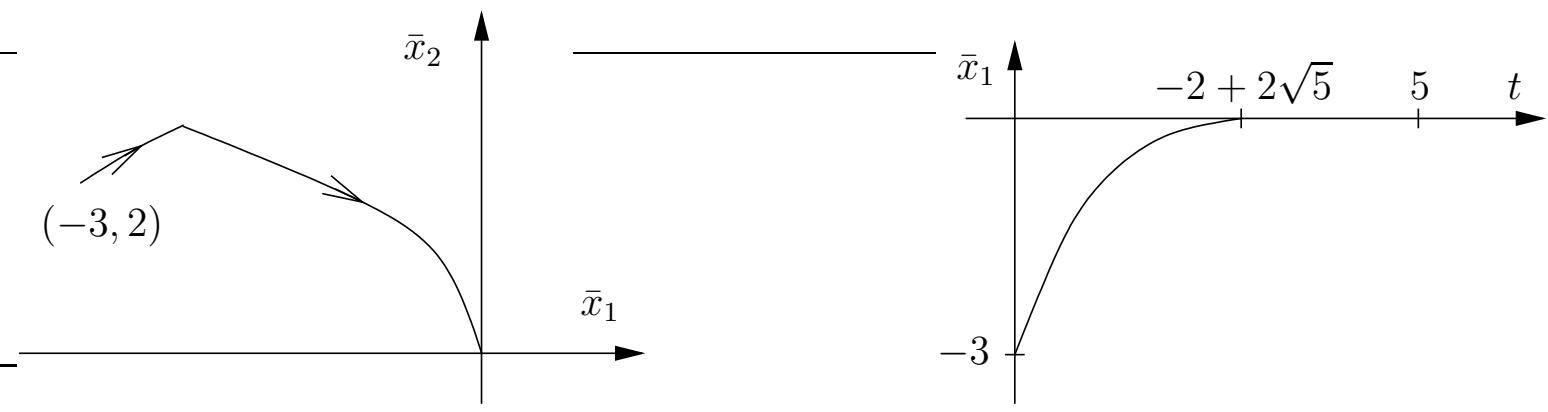

Figura 2.4: Um controle possível, mas não ótimo

O custo desta trajetória é aproximadamente 2, 2956, mas veremos a seguir que isso 
pode ser drasticamente reduzido. De qualquer maneira, esta trajetória é um indicativo de que possivelmente o controle ótimo será nulo em algum intervalo de tempo, ou, em outras palavras, que o controle ótimo terá um arco singular.

Suponha que $\bar{p}_{2}$ se anula um número finito de vezes e seja o $\left[t_{n-1}, t_{n}\right]$ o último intervalo, i.e., $\bar{x}_{1}\left(t_{n}\right)=\bar{x}_{2}\left(t_{n}\right)=\bar{p}_{1}\left(t_{n}\right)=\bar{p}_{2}\left(t_{n}\right)=\bar{p}_{2}\left(t_{n-1}\right)=0$. Nessas condições, denotando $\tau=t_{n}-t_{n-1}$, vale que

$$
\begin{aligned}
& 0= \pm \frac{\tau^{2}}{2}+\tau k_{1}+k_{2} \\
& 0= \pm \tau+k_{1} \\
& 0= \pm \frac{\tau^{3}}{6}+\frac{\tau^{2}}{2} k_{1}+\tau k_{2}+k_{3} \\
& 0= \pm \frac{\tau^{4}}{24}+\frac{\tau^{3}}{6} k_{1}+\frac{\tau^{2}}{2} k_{2}+\tau k_{3}
\end{aligned}
$$

de onde, necessariamente, $0=\tau=t_{n}-t_{n-1}$, absurdo. Logo, $\bar{p}_{2}$ se anula infinitas vezes. Como corolário, uma vez que o intervalo de tempo total é finito, os saltos no controle se acumulam.

Até agora já sabemos que a solução existe e é única, que o controle ótimo tem infinitas descontinuidades e possivelmente um arco singular. Uma tentativa tentadora é construir um controle baseado em (2.5.1), mas acrescentando saltos antes do arco singular. Claro que os pontos de descontinuidade serão diferentes dos de (2.5.1).

A sequência de singularidades precisam se acumular em algum ponto $0 \leq T_{a} \leq 5$, então uma boa tentativa é tomá-las em progressão geométrica.

Para facilitar, vamos considerar agora a mudança de coordenadas $s=t-T_{a}$. Veja a Figura 2.5. Então, queremos contruir uma sequência $\left\{s_{n}\right\}_{n \in \mathbb{N}}$ tal que $s_{n}=J s_{n-1}=$ $J^{n} s_{0}$, com $0<J<1$, de modo que $s_{n} \stackrel{n \rightarrow \infty}{\longrightarrow} 0$. Note que $-T_{a} \leq s_{n} \leq 0$, para todo $n \in \mathbb{N}$.

Desejamos que $\bar{p}_{2}\left(s_{n}\right)=0$, para todo $n \in \mathbb{N}$. Assim, como $\bar{p}_{2}$ é um polinômio de 

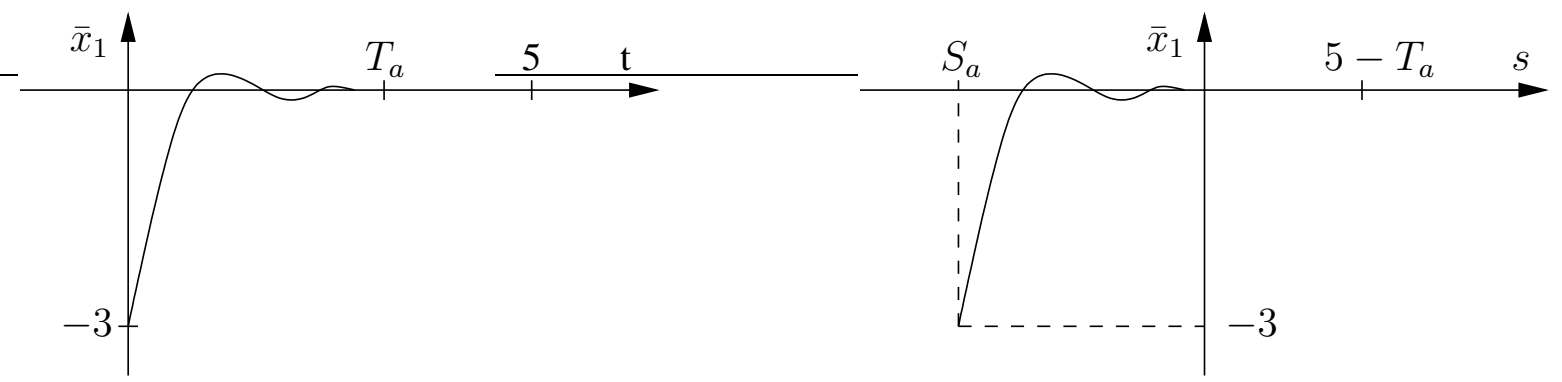

Figura 2.5: Componente $\bar{x}_{1}$ extremal procurado

grau 4 em cada intervalo $\left[s_{n}, s_{n+1}\right]$, devemos exigir que $\bar{p}_{2}(J s)=-J^{4} \bar{p}_{2}(s)$.

Denotando por $\bar{p}_{2}^{(i)}$ a i-ésima derivada de $\bar{p}_{2}$, sabemos das equações adjuntas que:

$$
\begin{array}{ll}
\bar{p}_{2}^{(1)}=-\bar{p}_{1} & \bar{p}_{2}^{(2)}=-\bar{x}_{1} \\
\bar{p}_{2}^{(3)}=-\bar{x}_{2} & \bar{p}_{2}^{(4)}=-\operatorname{sign}\left(\bar{p}_{2}\right) .
\end{array}
$$

Assim, suponha que $\left[s_{0}, s_{1}\right]$ é um intervalo onde $\bar{p}_{2}^{(4)}=-\operatorname{sign}\left(\bar{p}_{2}\right)=-1$ e que $S_{a}=-T_{a} \in\left[s_{0}, s_{1}\right]$. Então:

$$
\begin{gathered}
s_{0} \leq s \leq s_{1} \Rightarrow \bar{p}_{2}(s)=-\frac{s^{4}}{24}+A s^{3}+B s^{2}+C s+D \\
s_{n} \leq s \leq s_{n+1} \Rightarrow \bar{p}_{2}(s)=(-1)^{n}\left(-\frac{s^{4}}{24}+J^{n} A s^{3}+J^{2 n} B s^{2}+J^{3 n} C s+J^{4 n} D\right) .
\end{gathered}
$$

Como $\bar{p}_{2}$ e suas 3 derivadas são contínuas, obtemos:

$$
\begin{aligned}
& \bar{p}_{2}^{(1)}\left(s_{1}\right)=-\frac{s_{1}^{4}}{24}+A s_{1}^{3}+B s_{1}^{2}+C s_{1}+D=\frac{s_{1}^{4}}{24}-J A s_{1}^{3}-J^{2} B s_{1}^{2}-J^{3} C s_{1}-J^{4} D \\
& \bar{p}_{2}^{(2)}\left(s_{1}\right)=-\frac{s_{1}^{3}}{6}+3 A s_{1}^{2}+2 B s_{1}+C=\frac{s_{1}^{3}}{6}-3 J A s_{1}^{2}-2 J^{2} B s_{1}-J^{3} C \\
& \bar{p}_{2}^{(3)}\left(s_{1}\right)=-\frac{s_{1}^{2}}{2}+6 A s_{1}+2 B=\frac{s_{1}^{2}}{2}-6 J A s_{1}-2 J^{2} B \\
& \bar{p}_{2}^{(4)}\left(s_{1}\right)=-s_{1}+6 A=s_{1}-6 J A,
\end{aligned}
$$


de onde facilmente encontramos:

$$
\begin{array}{ll}
A=\frac{s_{1}}{3(1+J)} & B=-\frac{s_{1}^{2}}{2\left(1+J^{2}\right)} \\
C=\frac{s_{1}^{3}}{3\left(1+J^{3}\right)} & D=-\frac{s_{1}^{4}}{12\left(1+J^{4}\right)} .
\end{array}
$$

Como $\bar{p}_{2}\left(s_{1}\right)=0$, então $J$ é a única raíz real menor que 1 desse polinômio:

$$
J=\frac{3+\sqrt{33}-\sqrt{26+6 \sqrt{33}}}{4} \approx 0,2421 .
$$

Note que ainda não sabemos o valor de $s_{1}$, portanto ainda não conhecemos a sequência e tão pouco $\bar{p}_{2}$. Também desconhecemos $S_{a}$ e, consequentemente, $T_{a}$. Mas das condições iniciais do problema sabemos que:

$$
\begin{aligned}
-3 & =\bar{x}_{1}\left(S_{a}\right)=\frac{S_{a}^{2}}{2}-\frac{2 s_{1}}{1+J} S_{a}+\frac{s_{1}^{2}}{1+J^{2}} \\
2 & =\bar{x}_{2}\left(S_{a}\right)=S_{a}-\frac{2 s_{1}}{1+J} .
\end{aligned}
$$

Apesar do sistema acima dar dois possíveis valores para $S_{a}$, não há dúvida na escolha, pois apenas um deles é negativo. Com isso encontramos: $S_{a}=-T_{a} \approx-4,0714$ e $s_{1} \approx-3,7707$.

Assim, $\bar{p}_{2}$ está completamente descrito. Para cálculos, é útil escrever $n$ em função de $s$ :

$$
n(s)=\left\lfloor\frac{\ln (-s)-\ln \left(-s_{0}\right)}{\ln (J)}\right\rfloor .
$$

Dessa forma, acabamos de construir um extremal para o problema. Mas como a solução existe e é única, nosso extremal é a trajetória ótima. 
O custo total da solução ótima é:

$$
\begin{aligned}
C(\bar{x}, \bar{u}) & =\int_{0}^{5} \frac{\bar{x}_{1}^{2}(t)}{2} d t \\
& =\int_{0}^{T_{a}} \frac{\bar{x}_{1}^{2}(t)}{2} d t \\
& \approx 1,1331
\end{aligned}
$$

que de fato é muito menor do que aquele obtido pelo controle 2.5.1.

Observação 2.5.1. No seu artigo original, Fuller faz o tempo final ilimitado, mas neste texto seguimos de perto a apresentação feita por Marchal em [5] que fixa o tempo final.

\subsubsection{Um critério para verificar a presença do Fenômeno Fuller}

No exemplo anterior vimos que o Fenômeno Fuller é caracterizado pela acumulação das descontinuidades do controle ótimo. Muitas vezes essa acumulação ocorre em uma junção de um arco singular com um arco não singular, então um problema importante é ter critérios para caracterizar o controle nas proximidades de junções.

Em 1971 McDanell e Powers [6] obtiveram resultados que relacionam a continuidade das derivadas do controle na junção com a ordem do arco singular. Estes resultados dariam condições suficientes para verificar o Fenômeno Fuller em uma junção.

Mas em janeiro de 1980, Lewis [7] apresentou um contra-exemplo que mostrava que mais cuidado era necessário na definição de ordem, caso contrário os resultados de McDanell e Powers estariam incorretos. Isso levou a duas definições distintas de ordem: uma que Lewis chamou de ordem intrínsica e outra que ele chamou de ordem do arco singular.

Em dezembro de 1980, levando em consideração as observações de Lewis, Powers [8] ajustou os resultados que tinha apresentado junto com McDanell em 1971 e renomeou as definições que Lewis propôs, trocando "ordem intrínseca" por "ordem do problema". 
Infelizmente, a versão corrigida do teorema de McDanell e Powers exige que as duas ordens coincidam, fato que, em geral, não pode ser facilmente verificado.

Neste seção apresentamos as definições de ordem e os resultados obtidos em [6], já com as devidas correções. Utilizamos uma linguagem algébrica de modo a explicitar melhor os objetos com os quais lidamos e a evitar as usuais confusões acerca do assunto. Estaremos interessados apenas nas trajetórias extremais $(\bar{x}, \bar{u})$, então, para evitar uma notação sobrecarregada, vamos escrever apenas $(x, u)$.

Usaremos o Princípio do Mínimo de Pontryagin para tratar problemas da forma:

$$
\begin{aligned}
& \operatorname{minimizar} \int_{0}^{T_{f}(u)} f_{0}(x)+g_{0}(x) u \\
& \text { sujeito à }\left\{\begin{array}{l}
\dot{x}=f(x)+g(x) u \\
u:\left[0, T_{f}(u)\right] \rightarrow \mathbb{R} \\
|u(t)| \leq K(t) \\
x(0) \in A \\
x\left(T_{f}(u)\right) \in B
\end{array}, \forall t \in\left[0, T_{f}(u)\right]\right.
\end{aligned}
$$

onde $f, g, f_{0}$, e $g_{0}$ são analíticas com relação às duas variáveis e $K$ é analítica em uma vizinhança de cada junção.

Na forma sintética que utilizamos o problema fica: $P=\left(\Sigma, f_{0}(x)+g_{0}(x) u, A, B\right)$, $\operatorname{com} \Sigma=(\mathbb{R}, X, f(x)+g(x) u,\{u \in \operatorname{Mes}(\mathbb{R}):|u(t)| \leq K(t)\})$.

Como vamos utilizar o Princípio do Mínimo de Pontryagin, definimos a hamiltoniana do problema como:

$$
\begin{aligned}
H_{\lambda}(x, p, u) & =\langle p, f(x)+g(x) u\rangle+\lambda\left(f_{0}(x)+g_{0}(x) u\right) \\
& =\langle p, f(x)\rangle+\lambda f_{0}(x)+u\left(\langle p, g(x)\rangle+\lambda g_{0}(x)\right)
\end{aligned}
$$


Note que se definirmos:

$$
\begin{array}{ll}
\bar{f}=\left(f_{0}, f\right) & \bar{g}=\left(g_{0}, g\right) \\
\bar{x}=\left(x_{0}, x\right) & \bar{p}=(\lambda, p)
\end{array}
$$

onde $x_{0}$ satisfaz

$$
\dot{x}_{0}=f_{0}(x)+g_{0}(x) u,
$$

a hamiltoniana fica da forma $H_{\lambda}=\langle\bar{p}, \bar{f}\rangle+u\langle\bar{p}, \bar{g}\rangle$ e valem (lembre-se que $p$ é uma 1-forma, assim, sua representação matricial é um vetor linha):

$$
\begin{aligned}
& \dot{\bar{x}}=\frac{\partial H_{\lambda}}{\partial \bar{p}}=\bar{f}+\bar{g} u \\
& \dot{\bar{p}}=-\frac{\partial H_{\lambda}}{\partial \bar{x}}=-\bar{p} \frac{\partial f}{\partial x}-\bar{p} \frac{\partial g}{\partial x} u .
\end{aligned}
$$

Assim, para simplificar a notação desta seção, vamos cometer o abuso de denotar $\bar{f}$ por $f$. E, de maneira análoga, o mesmo abuso será cometido com relação a $g, x$ e $p$.

Como a hamiltoniana é linear em $u$, pelo Princípio do Bang-bang sabemos que nos arcos não singulares o controle é dado por $u(t)=\operatorname{sign}(\langle p(t), g(x(t))\rangle) K(t)$. Nos arcos singulares podemos calcular as derivadas com respeito a $t$ de $\langle p(t), g(x(t))\rangle$ até que $u$ apareça explicitamente e então resolver a equação $\frac{d^{k}}{d t^{k}}\langle p(t), g(x(t))\rangle=0$ em $u(t)$. Efetivar este cálculo é, no entando, mais complexo do que aparenta. Apresentamos a seguir alguns resultados nesse sentido.

Lema 2.5.2. Seja h um campo de vetores diferenciável. Então ao longo de um extremal vale a igualdade:

$$
\frac{d}{d t}\langle p, h\rangle=\langle p,[f, h]+u[g, h]\rangle .
$$


Demonstração. Sabemos que

$$
\frac{d}{d t}\langle p, h\rangle=\langle\dot{p}, h\rangle+\left\langle p, \frac{\partial h}{\partial x} \dot{x}\right\rangle .
$$

Usando as equações (2.5.2), a primeira parcela fica:

$$
\langle\dot{p}, h\rangle=\left\langle p,-\frac{\partial f}{\partial x} h\right\rangle+\left\langle p,-\frac{\partial g}{\partial x}\right\rangle u
$$

e a segunda parcela:

$$
\left\langle p, \frac{\partial h}{\partial x} \dot{x}\right\rangle=\left\langle p, \frac{\partial h}{\partial x} f\right\rangle+\left\langle p, \frac{\partial h}{\partial x} g\right\rangle u
$$

de onde segue o resultado.

Com este lema podemos calcular a primeira derivada com respeito a $t$ de $\langle p, g\rangle$, obtendo:

$$
\frac{d}{d t}\langle p, g\rangle=\langle p,[f, g]\rangle+u\langle p,[g, g]\rangle
$$

Da antissimetria do produto de Lie, $[g, g]=0$, portanto a primeira derivada de $\langle p, g\rangle$ nunca depende explicitamente de $u$. Assim, podemos continuar o processo de derivação:

$$
\frac{d^{2}}{d t^{2}}\langle p, g\rangle=\left\langle p, \operatorname{ad}_{f}^{2} g\right\rangle+u\left\langle p,\left[g, \operatorname{ad}_{f}^{1} g\right]\right\rangle
$$

Agora temos três possibilidades:

(i) $\left\langle p,\left[g, \operatorname{ad}_{f}^{1} g\right]\right\rangle=0$ para todo $x$ e todo $p$;

(ii) $\left\langle p,\left[g, \operatorname{ad}_{f}^{1} g\right]\right\rangle$ não é identicamente nulo, mas anula-se sobre a trajetória;

(iii) $\left\langle p,\left[g, \operatorname{ad}_{f}^{1} g\right]\right\rangle \neq 0$, inclusive sobre a trajetória. 
O primeiro e o terceiro caso são os mais simples: no primeiro caso, podemos continuar o processo de derivação independente da trajetória. No terceiro caso, o processo de derivação pára e encontramos uma expressão para o controle.

Já o segundo caso é mais delicado. A expressão da derivada dada pelo Lema 2.5.2 ainda vale, mas as técnicas exigem mais cuidado, pois já não temos mais liberdade para calcular derivadas com respeito a $t$ em outras direções que não a da trajetória. Basicamente, isso impossibilita conseguir mais informações sobre variações de trajetórias, adicionando muita dificuldade ao problema.

Essa diferença entre os casos motiva as definições de ordem. Mas antes de poder apresentá-las com o devido rigor, vamos a um lema que, com a discussão acima, é muito intuitivo.

Lema 2.5.3. Se as derivadas com relação a t até ordem $k$ de $\langle p, g\rangle$ têm o coeficiente de u nulo para todo $x$ e todo $p$, então $\left\langle p,\left[g, \mathrm{ad}_{f}^{i-1} g\right]\right\rangle=0$ para todo $x$ e todo $p$ e $\frac{d^{i}}{d t^{i}}\langle p, g\rangle=\left\langle p, \operatorname{ad}_{f}^{i} g\right\rangle, \operatorname{com} 1 \leq i \leq k$.

Demonstração. Provamos esta afirmação com indução em $i$. Evidentemente, vale para $i=1$. De fato, pelo Lema 2.5.2, temos que

$$
\frac{d}{d t}\langle p, g\rangle=\langle p,[f, g]\rangle+u\langle p,[g, g]\rangle
$$

Para o passo de indução, suponha que para algum $i<k$ vale que $\frac{d^{i}}{d t^{i}}\langle p, g\rangle=$ $\left\langle p, \operatorname{ad}_{f}^{i} g\right\rangle$. Aplicando o Lema (2.5.2), obtemos:

$$
\frac{d^{i+1}}{d t^{i+1}}\langle p, g\rangle=\left\langle p, \operatorname{ad}_{f}^{i+1} g\right\rangle+u\left\langle p,\left[g, \operatorname{ad}_{f}^{i} g\right]\right\rangle .
$$

Assim, como $i+1 \leq k$, sabemos por hipótese que o coeficiente de $u$ na expressão de $\frac{d^{i+1}}{d t^{2+1}}\langle p, g\rangle$ é nulo para todo $x$ e todo $p, \operatorname{logo}$ a tese vale para $i+1$. 
Corolário 2.5.4. Nas hipóteses do lema, $\left[g, \operatorname{ad}_{f}^{i-1} g\right]=0$ para todo $x$ e todo $1 \leq i \leq k$. Demonstração. Decorre direto da arbitrariedade de $p$.

Definição 2.5.5 (Ordem do problema). Seja $k$ o menor inteiro tal que $\left\langle p,\left[g, \operatorname{ad}_{f}^{k-1} g\right]\right\rangle$ não é identicamente nulo como função de $x$ e $p$. Defina $q=\frac{k}{2}$. Chamamos $q$ de ordem do problema. Se não existir tal $k$ dizemos que o problema tem ordem infinita.

Então, se $q$ é a ordem do problema, vale a seguinte igualdade:

$$
\frac{d^{2 q}}{d t^{2 q}}\langle p, g\rangle=\left\langle p, \operatorname{ad}_{f}^{2 q} g\right\rangle+u\left\langle p,\left[g, \operatorname{ad}_{f}^{2 q-1} g\right]\right\rangle
$$

Ainda assim, em um arco singular, essa equação não ajuda a determinar o controle, uma vez que $\left\langle p,\left[g, \mathrm{ad}_{f}^{k-1} g\right]\right\rangle$ pode se anular em subintervalos do arco singular. Isso leva a mais uma definição.

Definição 2.5.6 (Ordem do arco singular). Suponha que o problema tem ordem finita e que $(x, u)$ seja uma trajetória ótima sigular em $\left[t_{1}, t_{2}\right]$. Seja $k$ o menor inteiro tal que a derivada $\frac{d^{k}}{d t^{k}}\langle p, g\rangle$ tem alguma potência de u com um coeficiente não identicamente nulo sobre a trajetória no arco singular. Defina $s=\frac{k}{2}$. Chamamos $s$ de ordem do arco singular. Se o problema não tem ordem finita ou se nenhuma potência de u tem um coeficiente não identicamente nulo sobre a trajetória, dizemos que o arco tem ordem infinita.

Observe que a definição de ordem do problema difere da definição de ordem do arco singular, pois esta é calculada com respeito a uma trajetória. Por causa dessa diferença, em geral não é possível calcular a priori a ordem de um arco singular. Para ver um exemplo em que as ordens não são iguais veja [7] e [8].

Com frequência vamos nos referir a $\left\langle p, \operatorname{ad}_{f}^{2 q} g\right\rangle$ e a $\left\langle p,\left[g, \operatorname{ad}_{f}^{2 q-1} g\right]\right\rangle$, então é conveniente a seguinte notação: 
Notação 2.5.7. Se q a é ordem do problema então denotamos:

$$
\begin{aligned}
& A(x, p)=\left\langle p, \operatorname{ad}_{f}^{2 q} g\right\rangle \\
& B(x, p)=\left\langle p,\left[g, \operatorname{ad}_{f}^{2 q-1} g\right]\right\rangle
\end{aligned}
$$

e quando $A$ e $B$ estiverem calculados sobre uma trajetória:

$$
\begin{aligned}
& \alpha(t)=A(x(t), p(t)) \\
& \beta(t)=B(x(t), p(t)) .
\end{aligned}
$$

Implítico em sua definição está o fato da ordem do problema ser um número inteiro. Damos a seguir uma demonstração desse fato.

Proposição 2.5.8. A ordem do problema é sempre um número inteiro.

Antes de demonstrar essa proposição, vamos enunciar e demonstrar um lema.

Lema 2.5.9. Suponha que para algum $k$ inteiro $\left[g, \operatorname{ad}_{f}^{i} g\right]=0$ para todo $x$ e todo $p$, com $0 \leq i \leq k-1$. Então, se $j \in \mathbb{N}, 1 \leq j \leq k$

$$
\left[\operatorname{ad}_{f}^{j} g, \operatorname{ad}_{f}^{l} g\right]=-\left[\operatorname{ad}_{f}^{j-1} g, \operatorname{ad}_{f}^{l+1} g\right]
$$

para todo $x$ e todo $p$, com $0 \leq l \leq j$.

Demonstração. Provamos este lema por indução em $j$. Por hipótese, se $0 \leq l \leq k-1$, então $\left[g, \operatorname{ad}_{f}^{l} g\right]=0$, o que implica que $\left[f,\left[g, \operatorname{ad}_{f}^{l} g\right]\right]=0$. Aplicando a identidade de Jacobi nesta última igualdade, obtemos:

$$
\left[\operatorname{ad}_{f}^{1} g, \operatorname{ad}_{f}^{l} g\right]=-\left[g, \operatorname{ad}_{f}^{l+1} g\right]
$$


logo a tese é verdadeira para $j=1$.

Suponha a tese verdadeira até algum $j<k$. Então, se $0 \leq l \leq k-(j+1)$,

$$
\left[\operatorname{ad}_{f}^{m} g, \operatorname{ad}_{f}^{l+j-m} g\right]=0
$$

para todo $x$ e todo $p$ e $0 \leq m \leq j$. De fato, podemos fazer um indução em $m$. Se $m=0$, a equação (2.5.3) fica $\left[g, \operatorname{ad}_{f}^{l+j} g\right]$, que, como $l+j \leq k-1$, é nula por hipótese. Para o passo de indução, suponha a igualdade verdadeira para algum $m<j$. Note que valem as desigualdades:

$$
\begin{gathered}
m+1 \leq j \\
l+j-(m+1) \leq k-(m+1)
\end{gathered}
$$

então podemos usar a hipótese de indução em $j$ para concluir que:

$$
0=\left[\operatorname{ad}_{f}^{m} g, \operatorname{ad}_{f}^{l+j-m} g\right]=\left[\operatorname{ad}_{f}^{m+1} g, \operatorname{ad}_{f}^{l+j-(m+1)} g\right] .
$$

O que completa a indução em $m$.

Tomando $m=j$ na equação (2.5.3), temos que $\left[\operatorname{ad}_{f}^{j} g, \operatorname{ad}_{f}^{l} g\right]=0$, o que implica que $\left[f,\left[\operatorname{ad}_{f}^{j} g, \operatorname{ad}_{f}^{l} g\right]\right]=0$. Mais uma vez, pela identidade de Jacobi,

$$
\left[\operatorname{ad}_{f}^{j+1} g, \operatorname{ad}_{f}^{l} g\right]=-\left[\operatorname{ad}_{f}^{(j+1)-1} g, \operatorname{ad}_{f}^{l+1} g\right]
$$

concluindo a indução em $j$.

Corolário 2.5.10. Nas hipóteses do Lema 2.5.9, se $0 \leq j \leq k$, então

$$
\left[g, \operatorname{ad}_{f}^{k} g\right]=(-1)^{j}\left[\operatorname{ad}_{f}^{j} g, \operatorname{ad}_{f}^{k-j} g\right]
$$


Demonstração. Evidentemente, vale para $j=0$. Suponha que valha para algum $j<k$, então, como valem as desigualdades:

$$
\begin{gathered}
1 \leq j+1 \leq k \\
k-j-1=k-(j+1) \leq k-(j+1)
\end{gathered}
$$

estamos nas hipóteses do Lema 2.5.9. Portanto

$$
\left[g, \mathrm{ad}_{f}^{k} g\right]=(-1)^{j}\left[\operatorname{ad}_{f}^{j} g, \operatorname{ad}_{f}^{k-j} g\right]=(-1)^{j+1}\left[\operatorname{ad}_{f}^{j+1} g, \operatorname{ad}_{f}^{k-(j+1)} g\right] .
$$

Corolário 2.5.11. Nas hipóteses do Lema 2.5.9, se $k$ é par, então $\left[g, \operatorname{ad}_{f}^{k} g\right]=0$.

Demonstração. Basta notar que $\left[g, \operatorname{ad}_{f}^{k} g\right]=(-1)^{\frac{k}{2}}\left[\operatorname{ad}_{f}^{\frac{k}{2}} g, \operatorname{ad}_{f}^{\frac{k}{2}} g\right]$.

Agora fica simples provar a Proposição 2.5.8.

Demonstração da proposição 2.5.8. Suponha que até a derivada de ordem $k$ o controle tem um coeficiente identicamente nulo como função de $x$ e $p$. Observe que $k$ não é necessariamente a ordem do problema, mas menor ou igual a ela.

Do Lema 2.5.3, sabemos que $\left[g, \operatorname{ad}_{f}^{i} g\right]=0$, para todo $i=0, \ldots, k-1$. Então, se $k$ é par, e podemos aplicar o Corolário 2.5.11, concluindo que $\left[g, \operatorname{ad}_{f}^{k} g\right]=0$ para todo $x$ e todo $p$.

Mas, novamente pelo Lema 2.5.3, sabemos que $\left[g, \operatorname{ad}_{f}^{k} g\right]$ é justamente o coeficiente de $u$ da derivada de ordem $k+1$ de $\langle p, g\rangle$. Logo, a primeira derivada que $u$ tem um coeficiente não identicamente nulo não pode ser ímpar, portanto a ordem é sempre um número inteiro. 
Devido à sua continuidade, se $\beta$ não se anula em um instante $t$ então ela não se anula em toda uma vizinhança de $t$. Nesta vizinhança as ordens do problema e do arco singular coincidem. Se isso ocorre justamente em uma junção, então podemos estudá-la um pouco melhor. Para isso introduzimos uma nova noção de ordem.

Definição 2.5.12 (Ordem da junção). Dado um problema com ordem $q<\infty$ e uma trajetória com um arco singular e um arco não singular, e seja tc a junção destes arcos. Se $\beta\left(t_{c}\right) \neq 0$, dizemos que a junção tem ordem $q$.

Observação 2.5.13 (Condição de Legendre-Clebsch Generalizada). Em um arco sigular ótimo de ordem $s$ é necessário que:

$$
(-1)^{s} \beta \geq 0 .
$$

Essa condição é chamada de condição de Legendre-Clebsch generalizada ou, abreviadamente, condição GLC. Quando a desiguldade é estrita, chamamos de condição de Legendre-Clebsch generalizada estrita ou condição GLCS. Para uma prova deste resultado veja [9].

Evidentemente, em uma vizinhança de uma junção de ordem $q$, vale GLCS.

Finalmente podemos enunciar o teorema principal desta sessão. Nos será útil uma definição mais fraca e um tanto incomum de analiticidade por partes e uma definição de junção não analítica:

Definição 2.5.14 (Analiticidade por partes). Uma função real $f$ é dita analítica por partes em um intervalo $(a, b)$, se para cada $t_{c} \in(a, b)$, existe $t_{1} \in\left(a, t_{c}\right)$ e existe $t_{2} \in\left(t_{c}, b\right)$, tais que $f$ é analítica em $\left(t_{1}, t_{c}\right)$ e em $\left(t_{c}, t_{2}\right)$.

Definição 2.5.15 (Junção analítica). Uma junção é dita analítica se o controle ótimo é analítico por partes em alguma vizinhança da junção. 
Teorema 2.5.16. Seja $t_{c}$ uma junção analítica de ordem q. Se $r$ é o menor inteiro tal que $u^{(r)}$ é descontínuo em $t_{c}$, então $q+r$ é impar.

Demonstração. Por hipótese o controle é $\mathcal{C}^{r-1}$ na junção, então $\alpha$ e $\beta$ são de classe $\mathcal{C}^{r}$ em $t_{c}$.

Vamos denotar $\phi(t)=\langle p(t), g(x(t))\rangle$. Evidentemente, $\phi$ é de classe $\mathcal{C}^{2 q+r}$ em $t_{c}$, e como o controle é analítico por partes, $\phi$ é não nula numa vizinhança de $t_{c}$ interceptada pelo interior do arco não singular.

Tome $\epsilon \neq 0$ tal que $t_{c}+\epsilon$ é um ponto da intersecção dessa vizinhança com o interior do arco não singular e $t_{c}-\epsilon$ é um ponto do arco singular. Isso define uma bola de raio $|\epsilon|$ e centro $t_{c}$, então, para $\epsilon$ suficientemente pequeno, da definição de ordem da junção, sabemos que $\beta$ não se anula nessa bola e, além disso, vale GLCS estrita nessa vizinhança: $(-1)^{q} \beta>0$.

Sejam $u_{s}$ a restrição do controle $u$ ao arco singular e $u_{n}$ a restrição ao arco não singular, denotamos

$$
\begin{aligned}
& u_{s}^{(i)}\left(t_{c}\right)=\lim _{\epsilon \rightarrow 0} u^{(i)}\left(t_{c}-\epsilon\right) \\
& u_{n}^{(i)}\left(t_{c}\right)=\lim _{\epsilon \rightarrow 0} u^{(i)}\left(t_{c}+\epsilon\right) .
\end{aligned}
$$

Definimos $k=2 q+r$. Como $\phi^{(i)}$ é contínua em $t_{c}$ para $0 \leq i \leq k-1$ e $\phi \equiv 0$ no arco singular, a primeira parcela não nula da fórmula de Taylor de $\phi$ em torno de $t_{c}$ é a referente a $\phi^{(k)}$ :

$$
\phi\left(t_{c}+\epsilon\right)=\frac{\epsilon^{k}}{k !} \phi^{(k)}\left(t_{c}\right)+o\left(\epsilon^{k+1}\right) .
$$

Mas note que:

$$
\phi^{(k)}=\frac{d^{r} \phi^{2 q}}{d t^{r}}=\frac{d^{r}}{d t^{r}}(\alpha+\beta u),
$$


logo:

$$
\phi\left(t_{c}+\epsilon\right)=\frac{\epsilon^{k}}{k !}\left(\alpha^{(r)}+\sum_{i=0}^{r}\left(\begin{array}{l}
r \\
i
\end{array}\right) \beta^{(r-i)}\left(t_{c}\right) u_{n}^{(i)}\left(t_{c}\right)\right)+o\left(\epsilon^{k+1}\right) .
$$

Mas no arco singular

$$
0=\phi^{(2 q)}=\alpha+\beta u_{s} \Rightarrow \alpha=-\beta u_{s}
$$

de onde

$$
\alpha^{(r)}=-\sum_{i=0}^{r}\left(\begin{array}{l}
r \\
i
\end{array}\right) \beta^{(r-i)}\left(t_{c}\right) u_{s}^{(i)} .
$$

Substituindo na equação (2.5.4):

$$
\phi\left(t_{c}+\epsilon\right)=\frac{\epsilon^{k}}{k !}\left(\sum_{i=0}^{r}\left(\begin{array}{l}
r \\
i
\end{array}\right) \beta^{(r-i)}\left(t_{c}\right)\left(u_{n}^{(i)}\left(t_{c}\right)-u_{s}^{(i)}\left(t_{c}\right)\right)\right)+o\left(\epsilon^{k+1}\right)
$$

Mas como $u_{n}^{(i)}\left(t_{c}\right)=u_{s}^{(i)}\left(t_{c}\right)$ para $0 \leq i \leq r-1$, obtemos finalmente:

$$
\phi\left(t_{c}+\epsilon\right)=\frac{\epsilon^{k}}{k !} \beta\left(t_{c}\right)\left(u_{n}^{(r)}\left(t_{c}\right)-u_{s}^{(r)}\left(t_{c}\right)\right)+o\left(\epsilon^{k+1}\right) .
$$

Seja $\sigma=-\operatorname{sign}\left(\phi\left(t_{c}+\epsilon\right)\right)$, assim temos $u_{n}(t)=\sigma K(t)$, de onde $u_{n}^{(i)}\left(t_{c}\right)=\sigma K^{(i)}\left(t_{c}\right)$, $i=0, \ldots, r$. Agora considere a expansão:

$$
\begin{aligned}
\sigma K\left(t_{c}-\epsilon\right)-u\left(t_{c}-\epsilon\right) & =\sum_{i=0}^{r} \frac{(-\epsilon)^{i}}{i !}\left(\sigma K^{(i)}\left(t_{c}\right)-u_{s}^{(i)}\left(t_{c}\right)\right)+o\left(\epsilon^{r+1}\right) \\
& =\sum_{i=0}^{r} \frac{(-\epsilon)^{i}}{i !}\left(u_{n}^{(i)}\left(t_{c}\right)-u_{s}^{(i)}\left(t_{c}\right)\right)+o\left(\epsilon^{r+1}\right) \\
& =\frac{(-1)^{r} \epsilon^{r}}{r !}\left(u_{n}^{(r)}\left(t_{c}\right)-u_{s}^{(r)}\left(t_{c}\right)\right)+o\left(\epsilon^{r+1}\right) .
\end{aligned}
$$


de onde concluímos que

$$
u_{n}^{(r)}\left(t_{c}\right)-u_{s}^{(r)}\left(t_{c}\right)=\frac{(-1)^{r} r !}{\epsilon^{r}}\left(\sigma K\left(t_{c}-\epsilon\right)-u\left(t_{c}-\epsilon\right)\right)+o\left(\epsilon^{r+1}\right) .
$$

Substituindo esse resultado na equação (2.5.5), obtemos:

$$
\phi\left(t_{c}+\epsilon\right)=\frac{(-1)^{r} r ! \epsilon^{2 q}}{k !} \beta\left(t_{c}\right)\left(\sigma K\left(t_{c}-\epsilon\right)-u\left(t_{c}-\epsilon\right)\right)+o\left(\epsilon^{k+1}\right) .
$$

Lembrando que $\sigma$ foi definido tal que $\phi\left(t_{c}+\epsilon\right)>0 \Rightarrow \sigma=-1$ e $\phi\left(t_{c}+\epsilon\right)<0 \Rightarrow \sigma=1$, então

$$
(-1)^{r} \epsilon^{2 q} \beta\left(t_{c}\right)\left(K\left(t_{c}-\epsilon\right) \pm u\left(t_{c}-\epsilon\right)\right)<0 .
$$

Da condição GLCS em $t_{c}$ sabemos que $(-1)^{q} \beta\left(t_{c}\right)>0$. Então multiplicando a inequação (2.5.7) por esta quantidade positiva, obtemos:

$$
(-1)^{q+r} \epsilon^{2 q} \beta^{2}\left(t_{c}\right)\left(K\left(t_{c}-\epsilon\right) \pm u\left(t_{c}-\epsilon\right)\right)<0 .
$$

Como por hipótese $u^{(r)}$ é descontínua em $t_{c}$, sabemos que $u_{n}^{(r)}\left(t_{c}\right) \neq u_{s}^{(r)}\left(t_{c}\right)$. Portanto, da equação (2.5.6) e do fato de $u(t) \leq K(t)$, sabemos que $K\left(t_{c}-\epsilon\right) \pm u\left(t_{c}-\epsilon\right)>0$.

Assim, concluímos finalmente que $(-1)^{q+r}<0$. Logo, $q+r$ é ímpar.

Corolário 2.5.17. Se q é par e u analítica por partes, então o controle é contínuo em $t_{c}$.

Demonstração. Basta notar que $r \geq 1$.

Corolário 2.5.18. Se q é ímpar e u analítica por partes, então, ou o controle é descontínuo, ou tem a primeira derivada contínua em $t_{c}$.

Demonstração. Basta notar que ou $r=0$ ou $r \geq 2$. 
O teorema anterior exige que o controle seja analítico por partes em uma vizinhança da junção. Em geral essa exigência não é satisfeita, mas sob hipóteses mais fracas ainda podemos fazer afirmações sobre a continuidade do controle.

Teorema 2.5.19. Seja u um controle ótimo com arcos não singulares e singulares e $t_{c}$ uma junção de ordem q. São verdadeiras as afirmações:

(i) Se $\alpha\left(t_{c}\right) \pm \beta\left(t_{c}\right) K\left(t_{c}\right) \neq 0$, então u é descontínuo em $t_{c}$;

(ii) Se u é contínuo numa vizinhança de $t_{c}$ interceptada pelo interior do arco não singular, $\frac{d^{2 q}}{d t^{2 q}} \phi=0$ no arco não singular e $\beta\left(t_{c}\right) \neq 0$, então u é contínuo em $t_{c}$.

Demonstração. Lembre-se que em um arco singular $\frac{d^{2 q}}{d t^{2 q}} \phi=0$ e da definição de ordem da junção $\beta\left(t_{c}\right) \neq 0$. Então temos:

(i) Basta notar que $\alpha\left(t_{c}\right) \pm \beta\left(t_{c}\right) K\left(t_{c}\right) \neq 0=\alpha\left(t_{c}\right)+\beta\left(t_{c}\right) u_{s}\left(t_{c}\right)$. Como $\beta\left(t_{c}\right) \neq 0$, então $\left|u_{s}\left(t_{c}\right)\right| \neq K\left(t_{c}\right)=\left|u_{n}\left(t_{c}\right)\right|$. Logo, $u$ é descontínuo em $t_{c}$.

(ii) $\alpha\left(t_{c}\right)+\beta\left(t_{c}\right) u_{n}\left(t_{c}\right)=0=\alpha\left(t_{c}\right)+\beta\left(t_{c}\right) u_{s}\left(t_{c}\right) \Rightarrow u_{n}\left(t_{c}\right)=u_{s}\left(t_{c}\right)$. Portanto, o controle é contínuo em $t_{c}$.

Corolário 2.5.20. Nas hipóteses do teorema, se q é par e $\alpha\left(t_{c}\right) \pm \beta\left(t_{c}\right) K\left(t_{c}\right) \neq 0$, então a junção é não analítica.

Demonstração. Suponha por absurdo que a junção seja analítica, ou seja, que o controle seja analítico por partes em alguma vizinhança de $t_{c}$. Do Corolário 2.5.17, podemos concluir que o controle é contínuo na junção. Por outro lado, do item (i) do Teorema 2.5.19, o controle é descontínuo em $t_{c}$. Absurdo, logo a junção é não analítica.

Corolário 2.5.21. Nas hipóteses do teorema, se q é par, $\alpha \equiv 0, K\left(t_{c}\right) \neq 0$ e $\beta\left(t_{c}\right)>0$, então a junção é não analítica. 
Demonstração. Basta notar que nesse caso $\alpha\left(t_{c}\right) \pm \beta\left(t_{c}\right) K\left(t_{c}\right)= \pm \beta\left(t_{c}\right) K\left(t_{c}\right) \neq 0$.

Vejamos agora como esses resultados podem ajudar a identificar a presença do Fenômeno Fuller em uma junção. Para isso, utilizaremos o exemplo do carro, resolvido no início da seção.

Exemplo 2.5.22. Considere o problema $P=\left(\Sigma, \frac{x_{1}^{2}}{2},\{(-3,2)\},\{(0,0)\}, 5\right)$, com $\Sigma=$ $\left([-1,1], \mathbb{R}^{2},\left(x_{2}, u\right), \operatorname{Mes}([-1,1])\right)$. Já vimos que $\lambda=1$, então a hamiltoniana é dada por $H_{\lambda}(x, p, u)=p_{1} x_{2}+p_{2} u+\frac{x_{1}^{2}}{2}$ e as equações adjuntas por:

$$
(\operatorname{Adj}) \begin{cases}\dot{x}_{1}=x_{2} & \dot{x}_{2}=u \\ \dot{p}_{1}=-x_{1} & \dot{p}_{2}=-p_{1}\end{cases}
$$

Neste caso temos que $\phi=\frac{\partial H_{\lambda}}{\partial u}=p_{2}$ e então:

$$
\begin{array}{ll}
\phi^{(1)}=\dot{p}_{2}=-p_{1} & \phi^{(3)}=\dot{x}_{1}=x_{2} \\
\phi^{(2)}=-\dot{p}_{1}=x_{1} & \phi^{(4)}=\dot{x}_{2}=u .
\end{array}
$$

Assim, a ordem do problema é $q=2$ e como $\beta(t) \equiv 1$, a ordem do arco singular e a do problema coincidem. Então, como $\alpha(t) \equiv 0$, estamos nas condições do Corolário 2.5.21. Logo o controle não é analítico por partes.

No exemplo pudemos facilmente verificar que as ordens coincidiam por $\beta$ ser constante, mas esse não é o caso geral. Uma maneira de contornar essa dificuldade é tentar utilizar métodos numéricos para obter mais informações sobre o problema, como veremos na próxima seção. 


\subsection{Tratamento numérico de problemas de controle ótimo}

Como comentamos, não são poucas as dificuldades para se encontrar analiticamente um controle ótimo, pois dois pontos são ao mesmo tempo cruciais e quase sempre impossíveis de se fazer: integrar um sistema hamiltoniano e sintetizar o controle ótimo.

Mostraremos nesta seção uma possível abordagem numérica destes problemas. A idéia principal é recair no caso que os algorítimos de programação não linear estão preparados para lidar:

$$
\begin{aligned}
& \text { minimizar } g(x) \\
& \text { sujeito à }\left\{\begin{array}{l}
h(x)=0 \\
l \leq x \leq u
\end{array}\right.
\end{aligned}
$$

dados $g: \mathbb{R}^{n} \rightarrow \mathbb{R}, h: \mathbb{R}^{n} \rightarrow \mathbb{R}^{m}$ e $l, u \in \mathbb{R}^{n}$.

Observação 2.6.1. A maioria dos algorítmos de programação não linear podem tratar problemas ligeiramente mais gerais, onde as restrições $l \leq x \leq u$ são substituídas por $h_{1}(x) \leq 0$. Neste texto utilizaremos apenas o caso particular enunciado, porém não há dificuldade em estender os resultados para o caso geral.

\subsubsection{Método direto}

Dado um problema de controle ótimo $P=\left(\Sigma, c,\left\{x_{\text {inicial }}\right\},\left\{x_{\text {final }}\right\}\right)$, com

$$
\Sigma=\left([-1,1]^{m}, R^{n}, f, \operatorname{Mes}\left([-1,1]^{m}\right)\right),
$$

suponha que $(x, u)$ seja uma trajetória definida no intervalo $[0, T(u)]$. Tome uma partição $0=t_{0}<t_{1}<\ldots<t_{N-1}<t_{N}=T(u)$ tal que $t_{i+1}-t_{i}=\Delta t$ para todo $i=0, \ldots, N-1$. Suponha que o controle é constante em cada um destes intervalos, 
i.e., $u(t)=u_{i}$ para $t \in\left(t_{i}, t_{i+1}\right), i=0, \ldots, N-1$. Nos pontos $t_{i}$ o controle não precisa estar definido.

Denotando $x_{i}(t)=x(t)$ quanto $t \in\left[t_{i}, t_{i+1}\right]$, temos que em $\left(t_{i}, t_{i+1}\right)$ :

$$
\dot{x}_{i}=f\left(x_{i}, u_{i}\right)
$$

$\operatorname{com} x_{i}\left(t_{i}\right)=x_{i-1}\left(t_{i}\right), i=1, \ldots, N-1$, e $x_{0}(0)=x_{\text {inicial }}$.

Agora, escolhendo um método de integração numérica de passo único descrito por $\Phi$, temos:

$$
x_{i+1}\left(t_{i+1}\right)=x_{i}\left(t_{i+1}\right) \approx x_{i}\left(t_{i}\right)+\Phi\left(x_{i}\left(t_{i}\right), u_{i}\right) \Delta t .
$$

Assim, denotanto $x_{i}\left(t_{i}\right)$ apenas por $x_{i}$, podemos reescrever o problema de controle ótimo da seguinte maneira:

$$
\begin{aligned}
\operatorname{minimizar} & \sum_{i=0}^{N-1} c\left(x_{i}, u_{i}\right) \Delta t \\
\text { sujeito à } & \begin{cases}x_{i+1}-x_{i}-\Phi\left(x_{i}, u_{i}\right) \Delta t=0 & i=0, \ldots, N-1 \\
x_{0}-x_{\text {inicial }}=0 & \\
x_{N}-x_{\text {final }}=0 & i=0, \ldots, N-1, j=1, \ldots, m . \\
-1 \leq u_{i j} \leq 1 & \end{cases}
\end{aligned}
$$

Agora podemos utilizar qualquer algorítmo de programação não linear que lide bem com muitas variáveis para resolver numericamente este problema. Uma boa opção é utilizar alguma linguagem de modelagem matemática como $\mathrm{AMPL}^{1}$ ou $\mathrm{GAMS}^{2}$ e um

\footnotetext{
${ }^{1}$ AMPL - A Modeling Language for Mathematical Programming é uma linguagem de modelagem para problemas de otimização. Uma versão gratuita para estudantes pode ser obtida em http://www.ampl.com. Para utilizar o AMPL é necessário, além do ambiente, um solver, como os gratuitos IPOPT (sob a licensa CLP) e ALGENCAN (sob licensa GPL).

${ }^{2}$ GAMS - General Algebraic Modeling System, assim como AMPL, é um ambiente de otimização. Para utilizá-lo também é necessário instalar um solver. Muitos solvers estão disponíveis tanto em
} 
solver que implemente um algorítmo de ponto interior como o IPOPT ${ }^{3}[10]$ ou LOQO [11]. Pode-se também tentar utilizar métodos de Lagrangeano aumentado, como o ALGENCAN $^{4}$, referências [12] e [13].

A discretização proposta pode facilmente ser adaptada para problemas cujo espaço de parâmetros é mais complexo ou mesmo para integrações numéricas implícipas ou de passo múltiplo. Porém algum cuidado é necessário, uma vez que nesta abordagem o número de variáveis e restrições cresce muito conforme refinamos a partição.

Existem também alguns pacotes já preparados para lidar especificamente com problemas de controle ótimo. Um pacote muito utilizado é o NUDOCCCS. Outro pacote é o dynOpt ${ }^{5}$, que apesar de não termos testado muito, parece ser uma opção interessante. Ambos utilizam o método SQP para uma discretização completa do problema.

Em problemas de tempo ótimo, se soubermos a priori que o controle é puramente bang-bang, o TOS - Time Optimal Switching [14] é uma abordagem que pode gerar bons resultados.

\subsubsection{Aproximação das variáveis adjuntas}

Vimos como obter uma aproximação numérica para o estado e para o controle, mas muitas vezes, para análises pós-otimização, precisamos conhecer as variáveis adjuntas.

Uma boa aproximação das variáveis adjuntas são os multiplicadores de Lagrange do problema de programação não linear, no sentido de terem a mesma dinâmica. De fato, AMPL quanto em GAMS. Mais informações em http://www.gams.com.

${ }^{3} \mathrm{IPOPT}$ é um solver em $\mathrm{C}++$ sob licensa CPL, gratuito inclusive para uso comercial. A entrada de dados pode ser feita diretamente por código ou através de interfaces como AMPL

${ }^{4}$ ALGENCAN é um solver em Fortran 77 sob licensa GPL e como tal, gratuito inclusive para uso comercial.

${ }^{5}$ Mais informações em http://abs-5.me.washington.edu/. 
a lagrangeana do problema (2.6.1) é:

$$
\begin{aligned}
L(x, u, p, q)= & \sum_{i=0}^{N-1} c\left(x_{i}, u_{i}\right) \Delta t+\sum_{i=0}^{N-1}\left\langle p_{i+1}, x_{i+1}-x_{i}-\Phi_{i}\left(x_{i}, u_{i}\right) \Delta t\right\rangle+ \\
& \left\langle p_{0}, x_{0}-x_{\text {inicial }}\right\rangle+\left\langle p_{N+1}, x_{N}-x_{\text {final }}\right\rangle+ \\
& \sum_{i=0}^{N-1} \sum_{j=0}^{m}\left\langle q_{i j}, u_{i j}-1\right\rangle+\sum_{i=0}^{N-1} \sum_{j=0}^{m}\left\langle q_{(N+i) j},-u_{i j}-1\right\rangle
\end{aligned}
$$

então das condições KKT, veja referência [15], temos que $\nabla_{(x, u)} L=0$. Em particular,

$$
0=\frac{\partial L}{\partial x_{i}}=\frac{\partial c}{\partial x}\left(x_{i}, u_{i}\right) \Delta t+p_{i}+p_{i+1}\left(-I_{n}-\frac{\partial \Phi}{\partial x}\left(x_{i}, u_{i}\right) \Delta t\right)
$$

para $i=0, \ldots, N-1$ e $I_{n}$ a matriz identidade de dimensão $n$.

Note que as equações (2.6.2) são uma versão discreta das equações adjuntas. Com efeito, as equações adjuntas do problema são:

$$
\dot{p}=-p \frac{\partial f}{\partial x}+\frac{\partial c}{\partial x} .
$$

Então, supondo que $\Phi$ descreve um método consistente e que portanto $\Phi-f=$ $O\left(\Delta t^{d}\right)$, onde $d$ é a ordem do método, e utilizando a Fórmula de Taylor de $p$, obtemos:

$$
p_{k+1}=p_{k}-p_{k} \frac{\partial \Phi}{\partial x}\left(x_{k}, u_{k}\right) \Delta t+\frac{\partial c}{\partial x}\left(x_{k}, u_{k}\right) \Delta t+O\left(\Delta t^{2}\right)
$$

onde $p_{k}=p\left(t_{k}\right)$. 
Agora basta verificar que a relação descrita por (2.6.2) pode ser escrita como:

$$
\begin{aligned}
& p_{k}-p_{k+1} \frac{\partial \Phi}{\partial x}\left(x_{k}, u_{k}\right) \Delta t+ \frac{\partial c}{\partial x}\left(x_{k}, u_{k}\right) \Delta t= \\
&=p_{k}-\left(p_{k}-p_{k} \frac{\partial \Phi}{\partial x}\left(x_{k}, u_{k}\right) \Delta t+\frac{\partial c}{\partial x}\left(x_{k}, u_{k}\right) \Delta t+O\left(\Delta t^{2}\right)\right) \frac{\partial \Phi}{\partial x}\left(x_{k}, u_{k}\right) \Delta t+\frac{\partial c}{\partial x}\left(x_{k}, u_{k}\right) \Delta t= \\
&=p_{k}-p_{k} \frac{\partial \Phi}{\partial x}\left(x_{k}, u_{k}\right) \Delta t+\frac{\partial c}{\partial x}\left(x_{k}, u_{k}\right) \Delta t+O\left(\Delta t^{2}\right) \approx p_{k+1} .
\end{aligned}
$$

A última relação da expressão acima não é uma igualdade pois a constante de $O\left(\Delta t^{2}\right)$ desta equação e da equação anterior são, em geral, diferentes.

O cálculo dos multiplicadores de Lagrange, mesmo já conhecendo uma solução ótima, pode ser uma tarefa difícil, envolvendo muitas vezes inversões de matrizes de dimensão alta. Felizmente, a maior parte dos solvers fornece os multiplicadores na sua saída, o que torna simples a obtenção de uma aproximação das variáveis adjuntas. 


\section{Capítulo 3}

\section{Submarino automático}

Vamos analisar alguns aspectos do controle de veículos submarinos automáticos aplicando os estudos dos capítulos anteriores.

Estaremos interessados numa classe especial de submarinos: elipsoidais, submersos num fluido irrotacional, não viscoso, com volume infinito. Denotando por $x$ sua posição horizontal, consideramos seus movimentos restritos ao plano $(x, z)$ e descritos pelas equações:

$$
\left\{\begin{array}{l}
\dot{x}=v_{1} \cos \theta+v_{3} \sin \theta \\
\dot{z}=v_{3} \cos \theta-v_{1} \sin \theta \\
\dot{\theta}=\Omega \\
\dot{v}_{1}=-v_{3} \Omega \frac{m_{3}}{m_{1}}+\frac{u_{1}}{m_{1}} \\
\dot{v}_{3}=v_{1} \Omega \frac{m_{1}}{m_{3}}+\frac{u_{2}}{m_{3}} \\
\dot{\Omega}=v_{1} v_{3} \frac{m_{3}-m_{1}}{I}+\frac{u_{3}}{I}
\end{array}\right.
$$

onde $\theta$ é o angulo que o veículo está com relação ao eixo $x$ do referencial inercial, $\Omega$ a velocidade angular, $v_{1}$ e $v_{3}$ a velocidade horizontal e vertical do submarino com relação ao referencial móvel (com origem no centro de massa do submarino). O controle $u=\left(u_{1}, u_{2}, u_{3}\right)$ satisfaz $\left|u_{i}\right| \leq 1$, para $i=1,2,3$. As massas são tais que $m_{1}<m_{3}$ e $I$ 
é a inércia.

Em vários momentos será útil escrever esse sistema na forma $\dot{X}=f(X)+g_{1}(X) u_{1}+$ $g_{2}(X) u_{2}+g_{3}(X) u_{3}$, onde $X=\left(x, z, \theta, v_{1}, v_{2}, \Omega\right)$,

$$
f(X)=\left[\begin{array}{c}
v_{1} \cos \theta+v_{3} \sin \theta \\
v_{3} \cos \theta-v_{1} \sin \theta \\
\Omega \\
-v_{3} \Omega \frac{m_{3}}{m_{1}} \\
v_{1} \Omega \frac{m_{1}}{m_{3}} \\
v_{1} v_{3} \frac{m_{3}-m_{1}}{I}
\end{array}\right]
$$

e $g_{1}=\left(0,0,0, \frac{1}{m_{1}}, 0,0\right), g_{2}=\left(0,0,0,0, \frac{1}{m_{3}}, 0\right)$ e $g_{3}=\left(0,0,0,0,0, \frac{1}{I}\right)$.

A dedução deste modelo está além do âmbito deste trabalho e maiores detalhes podem ser obtidos em [16].

Nas próximas seções trataremos o problema de encontrar um controle que leve o submarino do estado $A=\left(x_{1}, z_{0}, 0,0,0,0\right)$ a um estado $B=\left(x_{2}, z_{0}, 0,0,0,0\right)$, no menor tempo possível. Começamos fazendo algumas observações interessantes deste sistema, passando ao tratamento numérico e finalizando com uma discussão sobre os indícios numéricos do aparecimento do Fenômeno Fuller.

\subsection{Trajetórias curiosamente não ótimas}

Desejamos ir de um ponto $A=\left(x_{1}, z_{0}, 0,0,0,0\right)$ a um ponto $B=\left(x_{2}, z_{0}, 0,0,0,0\right)$ no menor tempo possível. Naturalmente pensamos em percorrer o segmento de reta que liga esses dois pontos. Nossa intuição nos leva a considerar fortemente o controle que realiza esse percurso como o controle de tempo ótimo.

Vejamos o quanto a intuição nos ajudou. 
Para aplicar o Princípio do Máximo de Pontryagin, definimos

$$
H_{\lambda}=\langle p, f\rangle+\frac{p_{4}}{m_{1}} u_{1}+\frac{p_{5}}{m_{3}} u_{2}+\frac{p_{6}}{I} u_{3}-\lambda
$$

de onde concluímos que o controle ótimo é $u_{1}=\operatorname{sign}\left(p_{4}\right), u_{2}=\operatorname{sign}\left(p_{5}\right)$ e $u_{3}=\operatorname{sign}\left(p_{6}\right)$.

Supomos então que existe um controle ótimo tal que $\theta \equiv 0$. Fica fácil ver que nesse caso as equações adjuntas deste problema de controle ótimo ficam:

$$
\begin{array}{ll}
\dot{x}=v_{1} & \dot{p}_{1}=0 \\
\dot{z}=v_{3} & \dot{p}_{2}=0 \\
\dot{\theta}=0 & \dot{p}_{3}=-p_{1} v_{3}+p_{2} v_{1} \\
\dot{v}_{1}=\frac{u_{1}}{m_{1}} & \dot{p}_{4}=-p_{1}-p_{6} v_{3} \frac{m_{3}-m_{1}}{I} \\
\dot{v}_{3}=\frac{u_{2}}{m_{3}} & \dot{p}_{5}=-p_{2}-p_{6} v_{3} \frac{m_{3}-m_{1}}{I} \\
\dot{\Omega}=0 & \dot{p}_{6}=-p_{3}+p_{4} v_{3} \frac{m_{3}}{m_{1}}-p_{5} v_{1} \frac{m_{1}}{m_{3}}
\end{array}
$$

É importante notar que, quando o controle faz com que $\theta \equiv 0$, as componentes verticais e horizontais do movimento são desacopladas, de modo que as componente verticais do movimento não alteram o tempo final.

Assim, similarmente ao problema do carro, vemos que $u_{1}$ consiste necessariamente em acelerar o máximo até o ponto médio entre $A$ e $B$ e depois desacelerar ao máximo.

$\mathrm{E}$, como as movimentos verticais não alteram o tempo, temos liberdade para escolher $u_{2}$, desde que este sempre tome valores nos extremos do intervalo $[-1,1]$ e que $\left|u_{3}\right|=$ $\left|v_{1} v_{3}\left(m_{3}-m_{1}\right)\right| \leq 1$.

Podemos, por exemplo, mantê-lo nulo por um tempo, depois acelerar ao máximo por um curto intervalo de tempo, desacelerando ao máximo num intervalo de mesma 
duração e por fim permanecendo nulo:

$$
u_{2}(t)= \begin{cases}0, & 0 \leq t<t_{1} \\ 1, & t_{1} \leq t<t_{1}+\epsilon \\ -1, & t_{1}+\epsilon \leq t<t_{1}+2 \epsilon \\ 0, & t_{1}+2 \epsilon \leq t<t_{f} .\end{cases}
$$

Note que para $\epsilon$ suficientemente pequeno podemos garantir a desiguldade estrita $\left|u_{3}\right|=\left|v_{1} v_{3}\left(m_{3}-m_{1}\right)\right|<1$. Mas pelo Princípio do Máximo de Pontryagin, $u_{3}=$ $\operatorname{sign}\left(p_{6}\right), \log p_{6} \equiv 0$.

Mas então, $\dot{p}_{5}=-p_{2}$, ou seja, $p_{5}$ é um polinômio de grau 1 , de modo que $u_{2}$ pode ter no máximo uma descontinuidade. Isso contradiz a nossa escolha de $u_{2}$. Como tal escolha foi justificada considerando que existe um controle ótimo que mantém $\theta \equiv 0$, concluímos que não existe um controle ótimo tal que $\theta \equiv 0$.

Em particular, concluímos que o movimento em linha reta, quando considerado como o movimento tal que $\dot{z} \equiv 0$ e $\theta \equiv 0$, não é ótimo.

Este fato pode, num primeiro momento, parecer um tanto contra-intuitivo. Mas, se considerarmos que cada controle é um motor do submarino, vemos que estamos desprezando dois motores ao andar em linha reta. Seria muito melhor se pudessémos, por exemplo, usar o motor do torque $\left(u_{3}\right)$ para apontar instantaneamente a resultante das forças dos demais motores para o ponto $B$. Evidentemente não é possível fazer nenhuma ação instantânea, e, como mostrado em [16], um controle que primeiro rotaciona o submarino e depois anda em linha reta utilizando a força dos dois motores não é ótimo.

Observação 3.1.1. Um resultado semelhante pode ser ainda mais facilmente obtido para um sistema de controle mais simples (corpo rígido esférico), que num certo sentido 
é um caso particular do modelo acima. Considere o sistema:

$$
\left\{\begin{array}{l}
\dot{x}=v_{1} \cos \theta+v_{3} \sin \theta \\
\dot{z}=v_{3} \cos \theta-v_{1} \sin \theta \\
\dot{\theta}=\Omega \\
\dot{v}_{1}=\frac{u_{1}}{m} \\
\dot{v}_{3}=\frac{u_{2}}{m} \\
\dot{\Omega}=\frac{u_{3}}{I} .
\end{array}\right.
$$

A hamiltoniana fica:

$$
H_{\lambda}=p_{1}\left(v_{1} \cos \theta+v_{3} \sin \theta\right)+p_{2}\left(v_{3} \cos \theta-v_{1} \sin \theta\right)+p_{3} \Omega+\frac{p_{4}}{m} u_{1}+\frac{p_{5}}{m} u_{2}+\frac{p_{6}}{I} u_{3}-\lambda
$$

logo controle ótimo é $u_{1}=\operatorname{sign}\left(p_{4}\right), u_{2}=\operatorname{sign}\left(p_{5}\right)$ e $u_{3}=\operatorname{sign}\left(p_{6}\right)$.

Suponha que existe um controle ótimo tal que $\theta \equiv 0$

$$
\begin{aligned}
\dot{x} & =v_{1} & \dot{p}_{1} & =0 \\
\dot{z} & =v_{3} & \dot{p}_{2} & =0 \\
\dot{\theta} & =0 & \dot{p}_{3} & =-p_{1} v_{3}+p_{2} v_{1} \\
\dot{v}_{1} & =\frac{u_{1}}{m} & \dot{p}_{4} & =-p_{1} \\
\dot{v}_{3} & =\frac{u_{2}}{m} & \dot{p}_{5} & =-p_{2} \\
\dot{\Omega} & =0 & \dot{p}_{6} & =-p_{3} .
\end{aligned}
$$

Então, $p_{5}$ é um polinômio de grau 1 , $\log 0 u_{2}$ tem apenas uma descontinuidade. Mas como as componentes estão desacopladas, com uma argumento parecido com o anterior, podemos escolher $u_{2}$ com muito mais descontinuidades sem alterar o tempo total do 
percurso.

Claro que ainda é necessário que $u_{2}$ assuma valores nos extremos de $[-1,1]$ e que $\left|u_{3}\right|=\left|v_{1} v_{3}\left(m_{3}-m_{1}\right)\right| \leq 1$, mas não precisamos mais exigir a desiguldade estrita $\left|u_{3}\right|=\left|v_{1} v_{3}\left(m_{3}-m_{1}\right)\right|<1$, pois $p_{5}$ já não dependia de $p_{6}$.

\subsection{Simetrias do sistema}

Suponha sem perda de generalidade que queremos ir do estado $(-1,0,0,0,0,0)$ ao estado $(1,0,0,0,0,0)$. Suponha que $(X, u)$ seja uma trajetória definida em $[-T(u), T(u)]$ tal que $X(-T(u))=(-1,0,0,0,0,0)$ e $X(T(u))=(1,0,0,0,0,0)$. Podemos fazer a seguinte afirmação sobre esta trajetória:

Proposição 3.2.1. A componentes $x, \theta, v_{3}$ são impares e as componentes $z, v_{1}, \Omega$ são pares se e somente se $u_{1}$ e $u_{3}$ forem ímpares e $u_{2}$ for par.

Demonstração. Definindo $\tilde{X}=\left(\tilde{x}, \tilde{z}, \tilde{\theta}, \tilde{v}_{1}, \tilde{v}_{3}, \tilde{\Omega}\right)$, onde:

$$
\begin{aligned}
\tilde{x}(t) & =-x(-t) & \tilde{v}_{1}(t) & =v_{1}(-t) \\
\tilde{z}(t) & =z(-t) & \tilde{v}_{3}(t) & =-v_{3}(-t) \\
\tilde{\theta}(t) & =-\theta(-t) & \tilde{\Omega}(t) & =\Omega(-t)
\end{aligned}
$$


vemos que $\tilde{X}(T(u))=X(T(u))$ e que $\tilde{X}$ satisfaz $\dot{\tilde{X}}=f(\tilde{X})$. De fato,

$$
\begin{aligned}
\tilde{x}^{\prime}(t)=(-x(-t))^{\prime}=x^{\prime}(-t) & =v_{1}(-t) \cos (\theta(-t))+v_{3}(-t) \sin (\theta(-t)) \\
& =\tilde{v}_{1}(t) \cos (-\tilde{\theta}(t))-\tilde{v}_{3}(t) \sin (-\tilde{\theta}(t)) \\
& =\tilde{v}_{1}(t) \cos (\tilde{\theta}(t))+\tilde{v}_{3}(t) \sin (\tilde{\theta}(t)) \\
\tilde{z}^{\prime}(t)=(z(-t))^{\prime}=-z^{\prime}(-t) & =-v_{3}(-t) \cos (\theta(-t))+v_{1}(-t) \sin (\theta(-t)) \\
& =\tilde{v}_{3}(t) \cos (\tilde{\theta}(t))-\tilde{v}_{1}(t) \sin (\tilde{\theta}(t)) \\
& =\tilde{\Omega}^{\prime}(t) \\
\tilde{\theta}^{\prime}(t)=(-\theta(-t))^{\prime}=\theta^{\prime}(-t) & =\Omega(-t) \\
\tilde{v}_{1}^{\prime}(t)=\left(v_{1}(-t)\right)^{\prime}=-v_{1}^{\prime}(-t) & =v_{3}(-t) \Omega(-t) \frac{m_{3}}{m_{1}}-\frac{u_{1}(-t)}{m_{1}} \\
& =-\tilde{v}_{3}(t) \tilde{\Omega}(t) \frac{m_{3}}{m_{1}}-\frac{u_{1}(-t)}{m_{1}} \\
& =\tilde{v}_{1}(t) \tilde{v}_{3}(t) \frac{m_{3}-m_{1}}{I}-\frac{u_{3}(-t)}{I} \\
\tilde{v}_{3}^{\prime}(t)=\left(-v_{3}(-t)\right)^{\prime}=v_{3}^{\prime}(-t) & =v_{1}(-t) \Omega(-t) \frac{m_{1}}{m_{3}}+\frac{u_{2}(-t)}{m_{3}} \\
& =\tilde{v}_{1}(t) \tilde{\Omega}(t) \frac{m_{1}}{m_{3}}+\frac{u_{2}(-t)}{m_{3}} \\
\tilde{\Omega}^{\prime}(t)=(\Omega(-t))^{\prime}=-\Omega^{\prime}(-t) & =-v_{1}(-t) v_{3}(-t) \frac{m_{3}-m_{1}}{I}-\frac{u_{3}(-t)}{I} \\
& \\
&
\end{aligned}
$$

Assim, pelo teorema de unicidade, decorre a proposição.

\subsection{Resultados numéricos}

Implementamos em AMPL o problema de ir do estado $(0,1,0,0,0,0)$ ao estado $(2,1,0,0,0,0)$ como descrito na Seção 2.6, utilizando o solver IPOPT e o Método de Heun para a integração numérica com $2 \times 10^{3}$ passos. Os valores das constantes utilizadas são $m_{1}=13,2, m_{2}=25,6$ e $I=0,12$, como em [17]. 
O tempo mínimo obtido foi 9,25485. Veja nas Figuras 3.1, 3.2 e 3.3 os gráficos dos resultados numéricos.
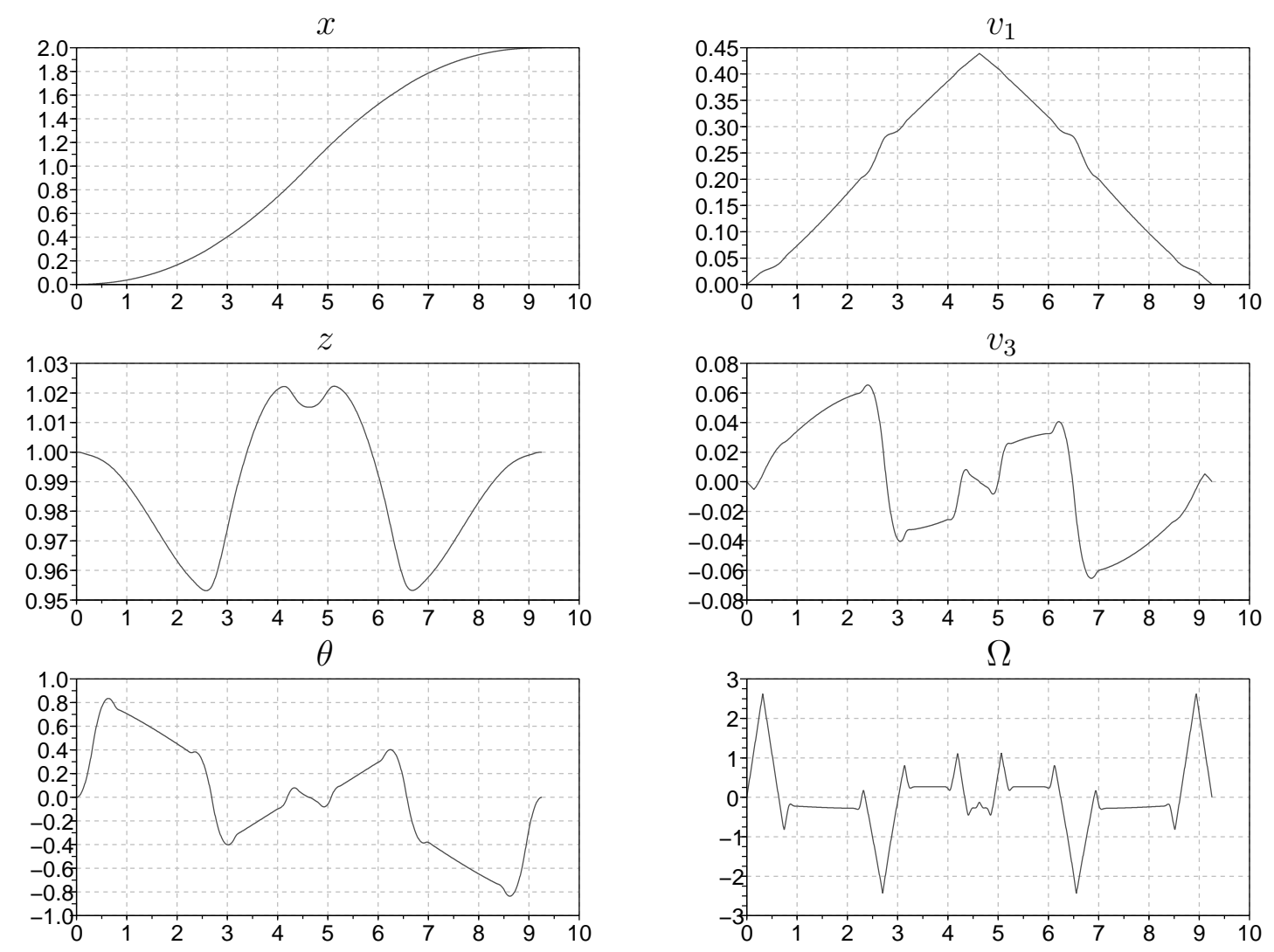

Figura 3.1: Órbita ótima

Observando estes resultados percebemos fortes indícios da existência de arcos singulares do controle $u_{3}$ e de chattering nas junções.

A presença do Fenômeno Fuller nestas junções não é surpreendente pois, como $u_{1}$ e $u_{2}$ são constantes em uma vizinhança das junções de $u_{3}$, podemos calcular analiticamente a ordem do problema com relação a $u_{3}$, obtendo $q=2$. Isto quer dizer que

$$
\frac{d^{4}}{d t^{4}}\langle p, g\rangle=\left\langle p, \operatorname{ad}_{f}^{4} g\right\rangle+\left\langle p,\left[g, \operatorname{ad}_{f}^{3} g\right]\right\rangle u_{3}
$$

$\operatorname{com}\left\langle p,\left[g, \operatorname{ad}_{f}^{3} g\right]\right\rangle$ não identicamente nulo. 

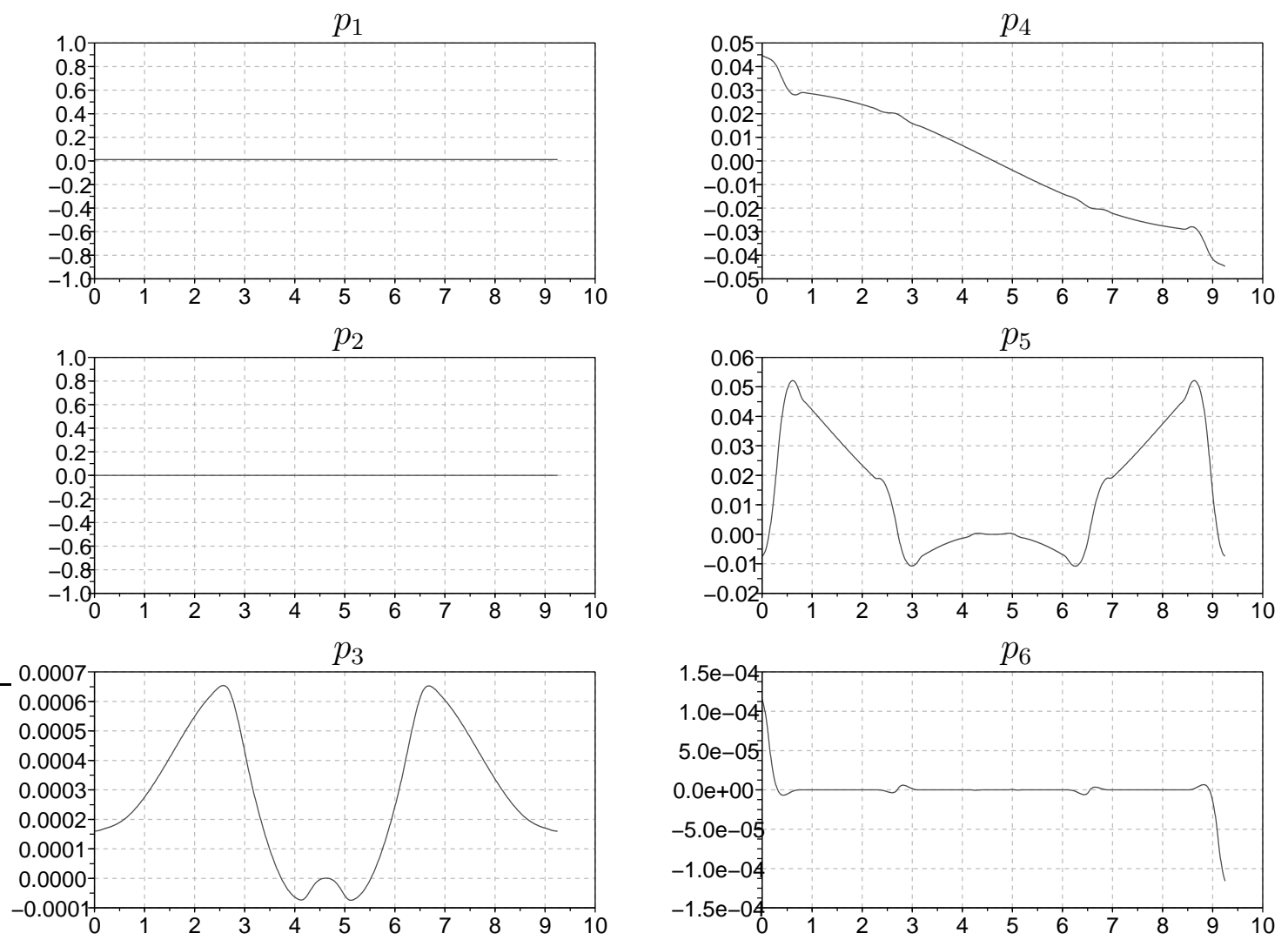

Figura 3.2: Variáveis adjuntas 

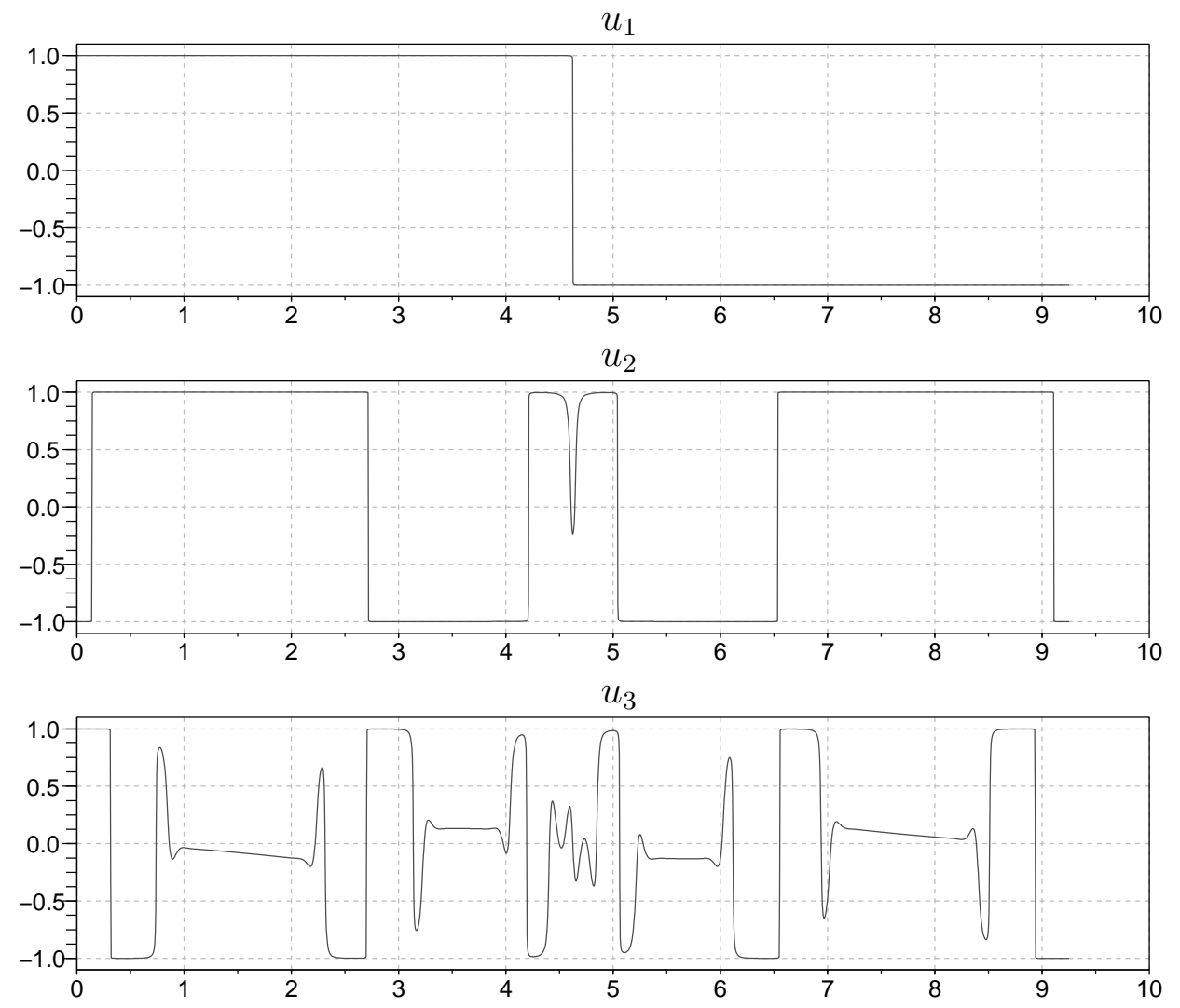

Figura 3.3: Controle ótimo 
Agora, utilizando os resultados numéricos, podemos calcular $\left\langle p,\left[g, \operatorname{ad}_{f}^{3} g\right]\right\rangle$ sobre a trajetória ótima verificando que nunca se anula, como pode ser visto na Figura 3.4. Assim, toda junção tem ordem 2 e vale a condição GLCS em todo o intervalo.

Note que, nos arcos singulares, $u_{3}$ parece tomar valores no interior de $[-1,1]$ e com sinal oposto ao do valor que assumia no arco não singular, sugerindo uma descontinuidade do controle nas junções. Essa sugestão é reforçada pelo item (i) do Teorema 2.5.19. De fato, aparentemente $u_{3}$ é contínuo no interior do arco singular e podemos ver na Figura 3.5 que $\alpha \pm \beta \neq 0$ em todo o intervalo, de modo que o controle é descontínuo nas junções. Quando for este o caso, de acordo com o Corolário 2.5.17, $u_{3}$ não pode ser analítico por partes, implicando a existência de Fenômeno Fuller nestas junções.

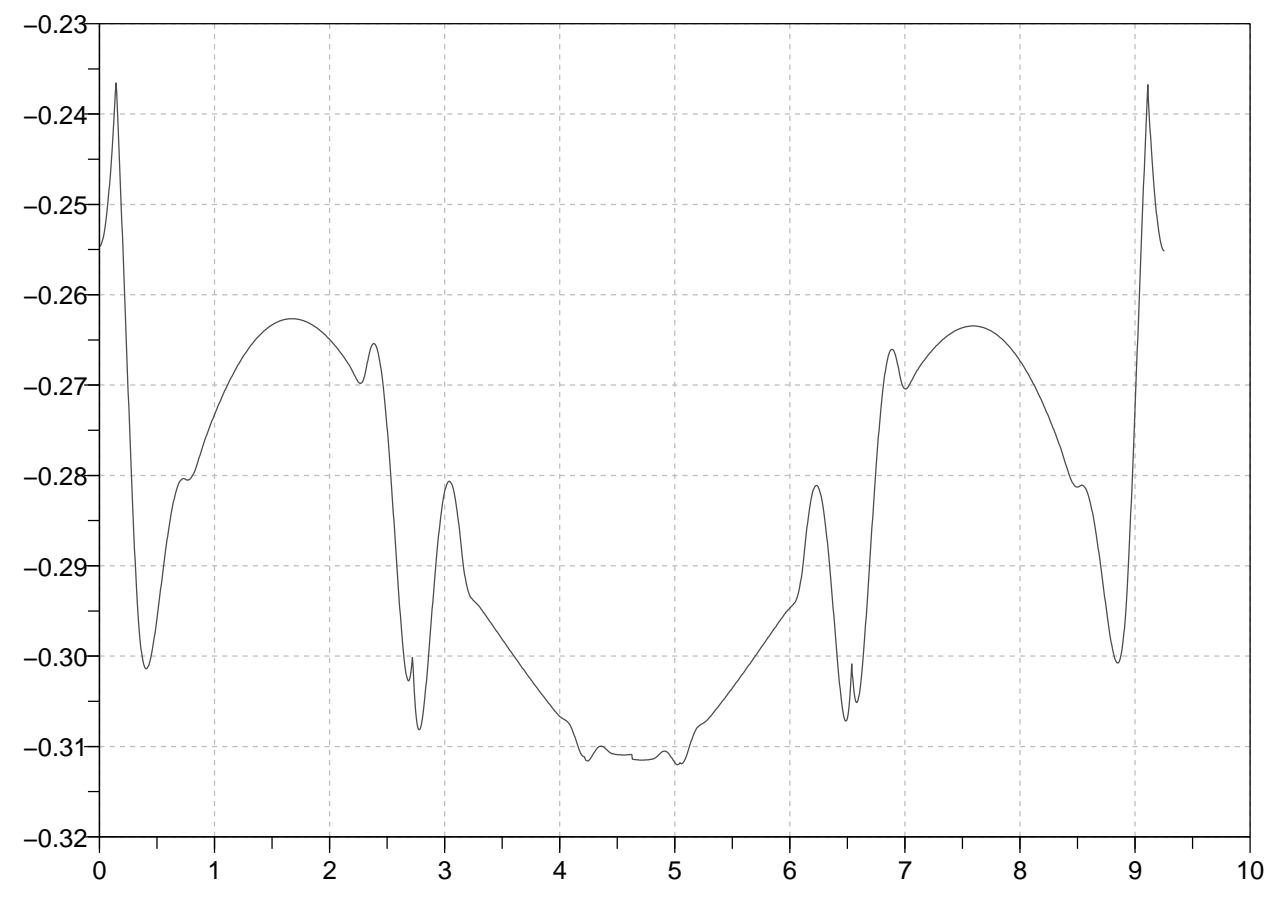

Figura 3.4: Função $\beta$ 

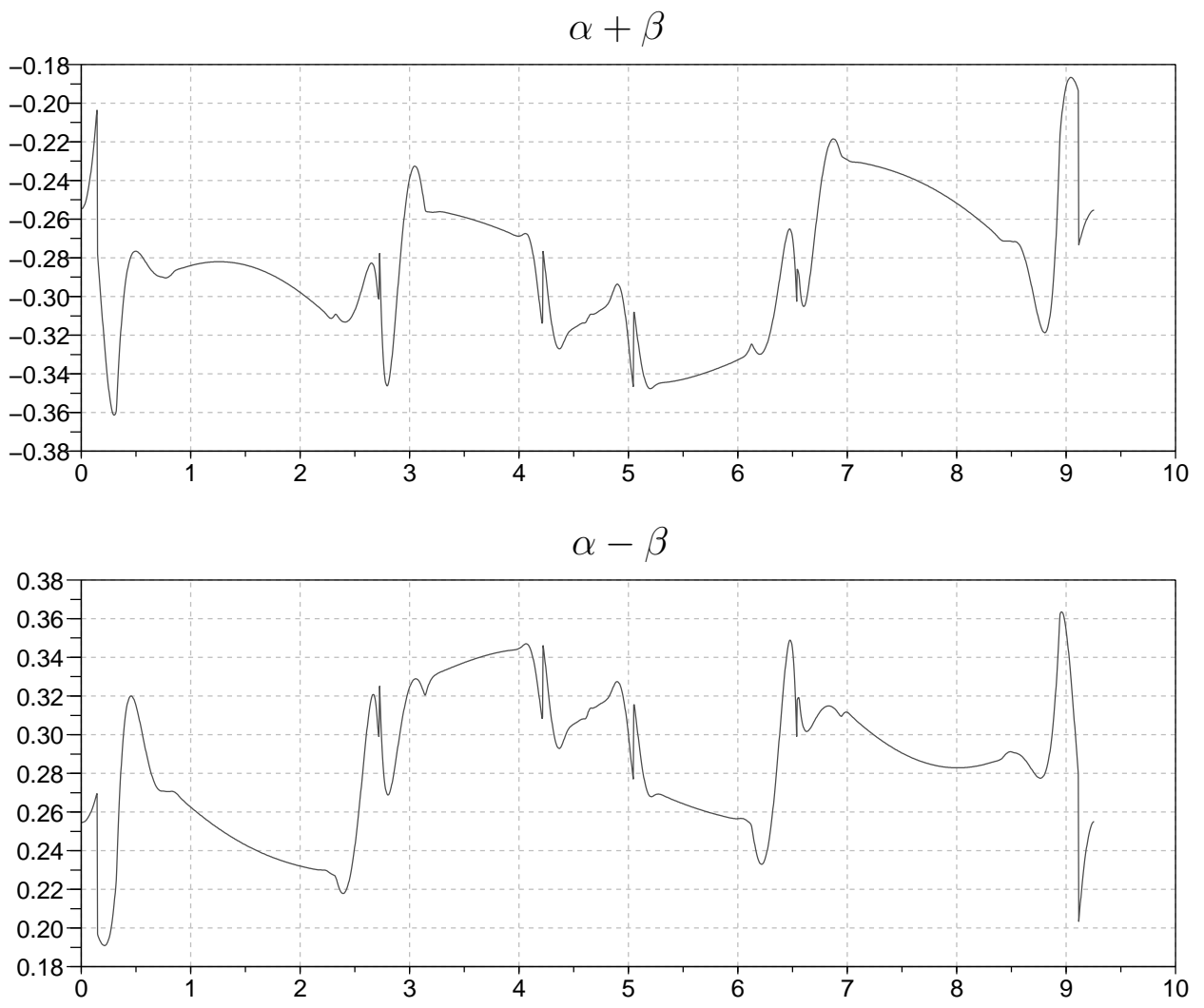

Figura 3.5: Gráfico de $\alpha \pm \beta$ 


\section{Capítulo 4}

\section{Conclusões}

O Princípio do Máximo de Pontryagin é uma ferramenta poderosa, muita informação sobre as soluções dos problemas de controle ótimo pode ser obtida com ele. Em particular, o Princípio do Bang-bang fornece informação valiosa sobre o controle nos arcos não singulares.

Porém, encontrar soluções analíticas é quase sempre impossível por diversos motivos. Dentre muitos, podemos ressaltar a necessidade de integrar campos hamiltonianos, a falta de critérios para encontrar o controle em arcos singulares cuja ordem difere da do problema e a possível presença do Fenômeno Fuller, que dificulta a síntese do controle.

Para tentar contornar estas dificuldades em um problema aplicado, apresentamos uma abordagem numérica. Nesta abordagem, vimos a interessante relação entre os multiplicadores de Lagrange do KKT e as variáveis adjuntas do Princípio do Máximo de Pontryagin.

Quando aplicamos esta abordagem numérica ao veículo autônomo subaquático, conseguimos, com base na teoria estudada anteriormante, inferir sobre caracteríscas deste sistema, encontrando indícios numéricos de que o problema é singular e que possivelmente o Fenômeno Fuller está presente nas junções. 


\section{Apêndice A}

\section{Listagem dos programas}

Neste apêndice listamos todos os códigos fonte de programas ou scripts utilizados para o tratamento numérico do submarino automático do Capítulo 3.

Estes códigos são para simples referência e questões sofisticadas de desenvolvimento, como eficiência e reaproveitabilidade, não são tratadas por não pertencerem ao escopo deste trabalho.

\section{A.1 Programa para cálculo da ordem do problema}

Este é um programa para Maxima ${ }^{1}$ que permite calcular a ordem do problema, $q$, com relação à $k$-ésima componente do controle, considerando que as demais componentes são constantes.

Adicionalmente, retorna $A$ e $B$ como na Notação 2.5.7.

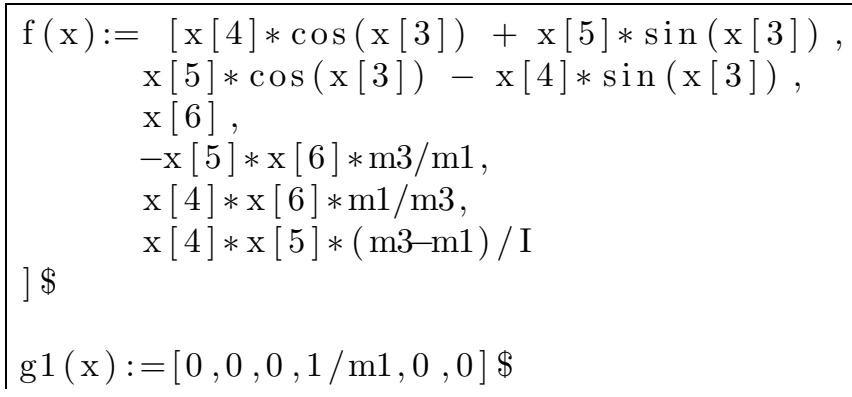

${ }^{1}$ O Máxima é uma ferramenta de matemática simbólica distribuido sob licensa GPL e como tal gratuito para uso, inclusive comercial. Mais informações em http://maxima.sourceforge.net/. 


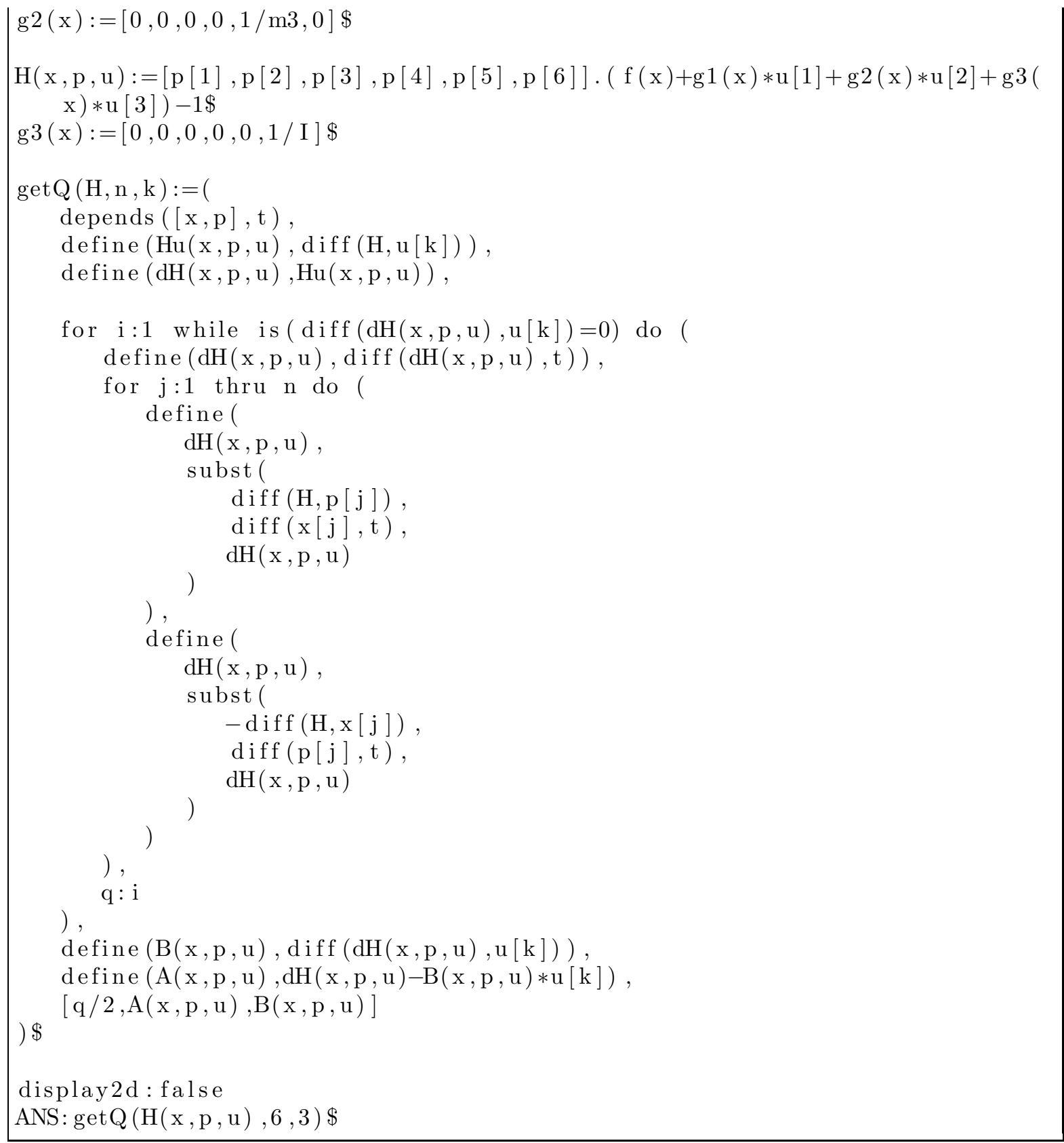

Listagem A.1: calcorder.wxm

\section{A.2 Scripts AMPL}

Estes quatro scripts utilizam AMPL/IPOPT para obter uma solução numérica do problema do submarino automático implementado a abordagem numérica descrita na Seção 2.6. O problema é discretizado com o método de Heun. 


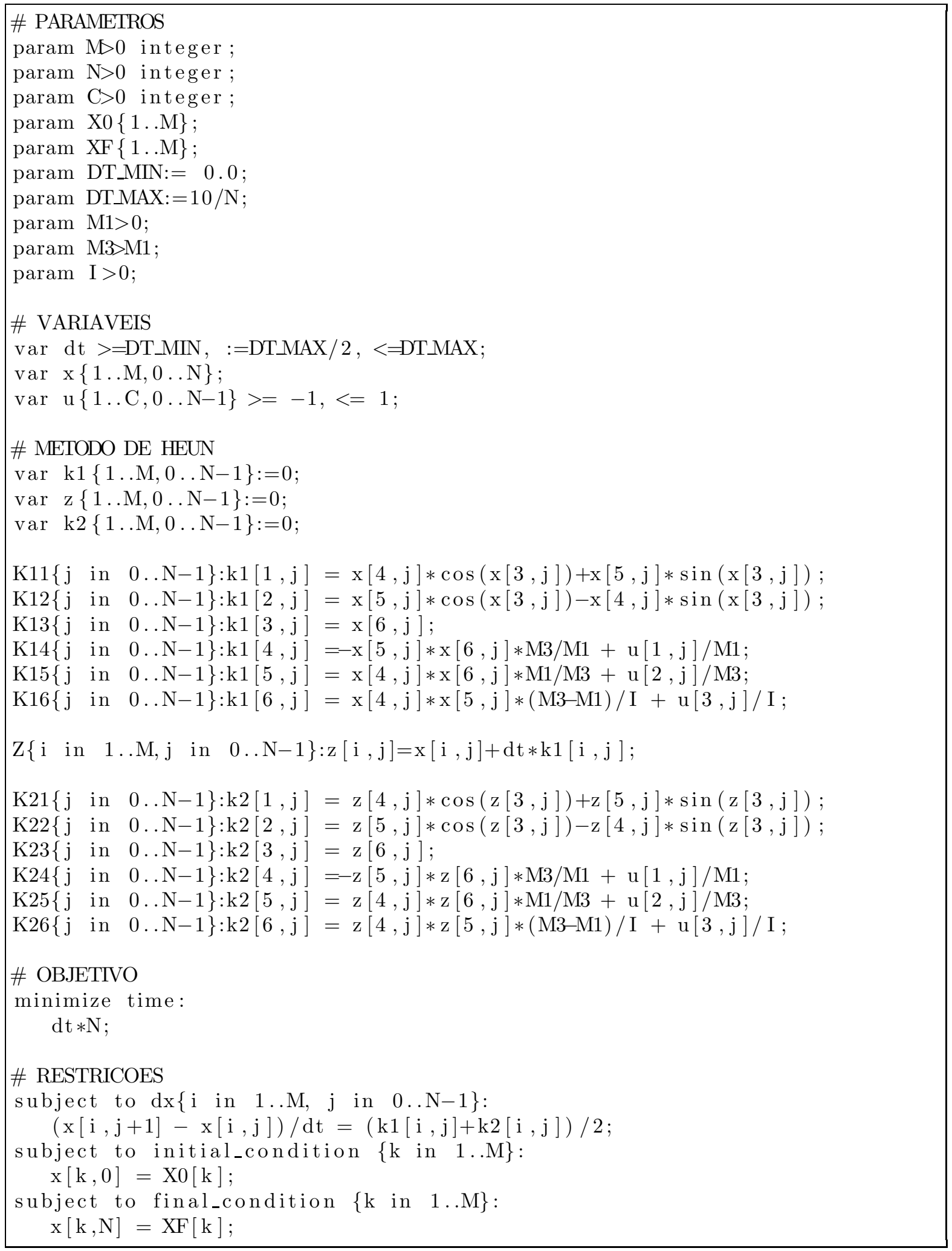

Listagem A.2: auv.mod 


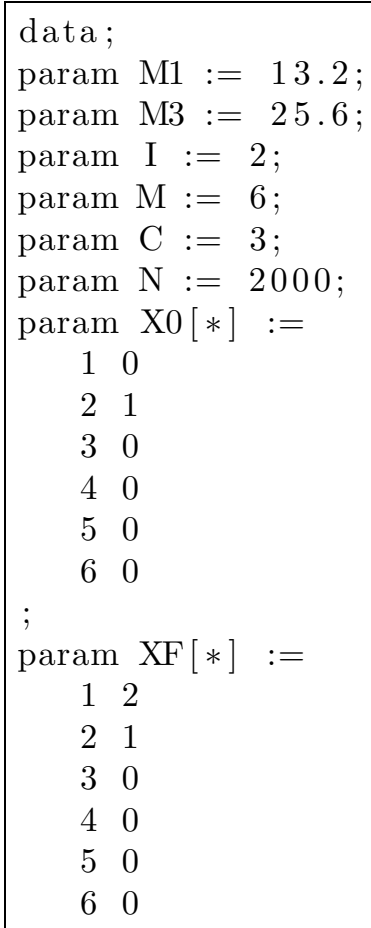

Listagem A.3: auv.dat

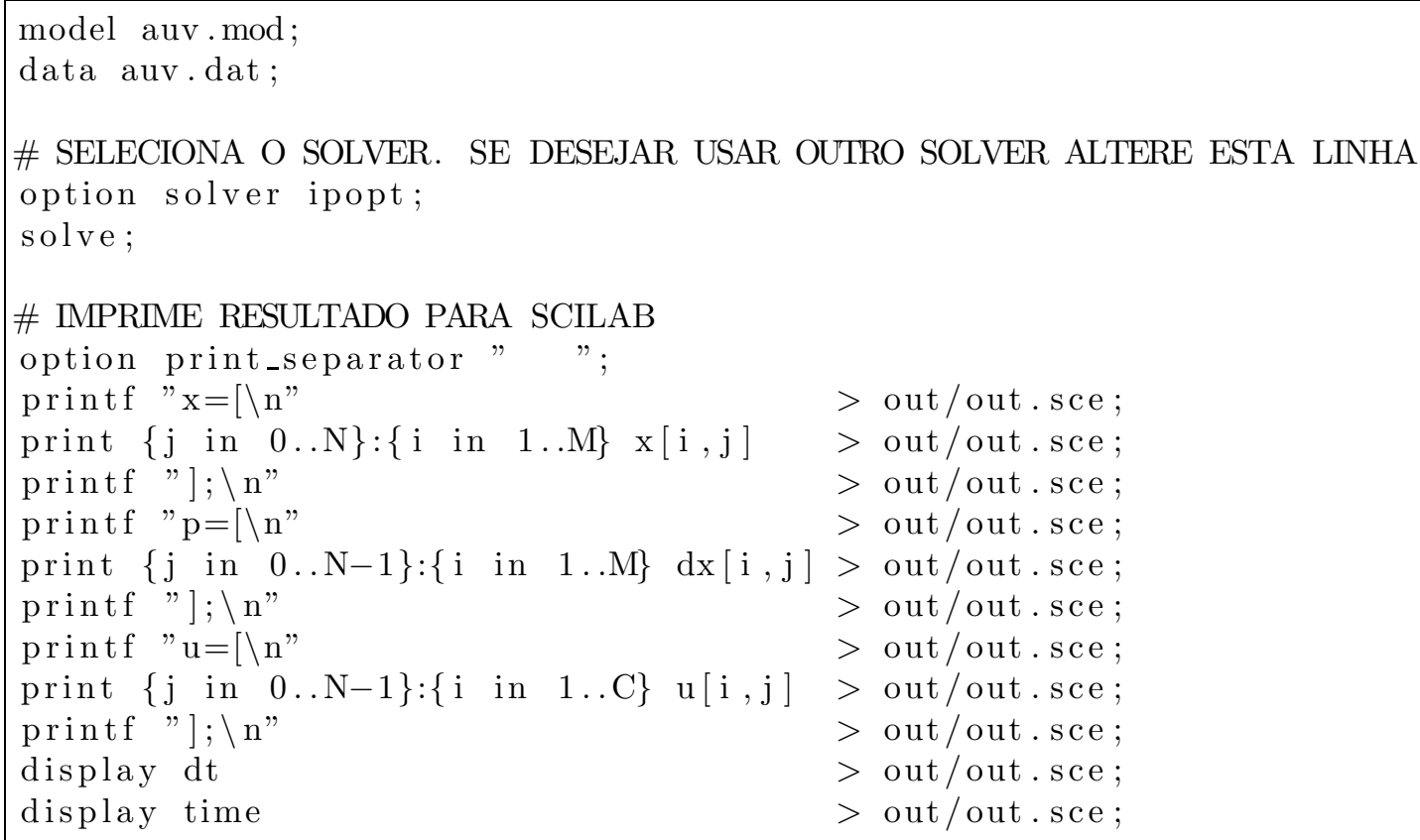

Listagem A.4: auv.run 


\section{Referências Bibliográficas}

[1] KUPKA, I. Introduction to the theory of systems. 1. ed. Brasil: IMPA, 1986. 123 p.

[2] PONTRYAGIN, L. S. et al. Mathematical Theory of Optimal Processes (Classics of Soviet Mathematics). [S.l.]: Gordon and Breach Science, 1962.

[3] BERKOVITZ, L. D. Optimal Control Theory. 1. ed. EUA: Sringer-Verlag, 1924. $304 \mathrm{p}$.

[4] KUPKA, I. A. K. The ubiquity of fuller's phenomenon. In: Nonlinear controllability and optimal control. New York: Dekker, 1990, (Monogr. Textbooks Pure Appl. Math., v. 133). p. 313-350.

[5] MARCHAL, C. Chattering arcs and chattering controls. J. Optimization Theory Appl., v. 11, p. 441-468, 1973. ISSN 0022-3239.

[6] MCDANELL, J. P.; POWERS, W. F. Necessary conditions for joining optimal singular and nonsingular subarcs. SIAM J. Control, v. 9, p. 161-173, 1971. ISSN 1095-7138.

[7] LEWIS, R. M. Definitions of order and junction conditions in singular optimal control problems. SIAM J. Control Optim., v. 18, n. 1, p. 21-32, 1980. ISSN 00361402.

[8] POWERS, W. F. On the order of singular optimal control problems. J. Optim. Theory Appl., v. 32, n. 4, p. 479-489, 1980. ISSN 0022-3239. 
[9] ROBBINS, H. M. A generalized legendre-clebsch condition for the singular cases of optimal control. IBM J. Res. Develop., v. 11, n. 4, p. 361-372, 1967.

[10] WÄCHTER, A.; BIEGLER, L. T. On the implementation of an interior-point filter line-search algorithm for large-scale nonlinear programming. Math. Program., v. 106, n. 1, Ser. A, p. 25-57, 2006. ISSN 0025-5610.

[11] VANDERBEI, R. J.; SHANNO, D. F. An interior-point algorithm for nonconvex nonlinear programming. Comput. Optim. Appl., v. 13, n. 1-3, p. 231-252, 1999. ISSN 0926-6003. Computational optimization - a tribute to Olvi Mangasarian, Part II.

[12] ANDREANI, R. et al. On augmented Lagrangian methods with general lower-level constraints. SIAM J. Optim., v. 18, n. 4, p. 1286-1309, 2007. ISSN 1052-6234.

[13] ANDREANI, R. et al. Augmented Lagrangian methods under the constant positive linear dependence constraint qualification. Math. Program., v. 111, n. 1-2, Ser. B, p. 5-32, 2008. ISSN 0025-5610.

[14] KAYA, C. Y.; NOAKES, J. L. Computational method for time-optimal switching control. J. Optim. Theory Appl., v. 117, n. 1, p. 69-92, 2003. ISSN 0022-3239.

[15] MARTINEZ, J. M. Métodos computacionais de otimização. 1. ed. Rio de Janeiro: IMPA, 1995. $256 \mathrm{p}$.

[16] CHYBA, M.; LEONARD, N. E.; SONTAG, E. D. Optimality for underwater vehicles. Decision and Control. Proc. 40th IEEE Conference on, v. 5, p. 4204-4209, 2001.

[17] CHYBA, M.; SUSSMANN, H.; MAURER, G. V. H. Underwater vehicles: the minimum time problem. Decision and Control. Proc. 43rd IEEE Conference on, v. 2, p. 1370-1375, 2004. ISSN 0191-2216. 
[18] FOURER, R.; GAY, D. M.; KERNIGHAN, B. W. AMPL: A modeling language for mathematical programming. 1. ed. EUA: Boyd \& Fraser, 1992. 351 p.

[19] BÜSKENS, C.; MAURER, H. SQP-methods for solving optimal control problems with control and state constraints: adjoint variables, sensitivity analysis and real-time control. J. Comput. Appl. Math., v. 120, n. 1-2, p. 85-108, 2000. ISSN 0377-0427. SQP-based direct discretization methods for practical optimal control problems.

[20] BÜSKENS, C.; MAURER, H. *Sensitivity analysis and real-time control of parametric optimal control problems using nonlinear programming methods. In: Online optimization of large scale systems. Berlin: Springer, 2001. p. 57-68.

[21] CHYBA, M. Underwater vehicles: a surprising non time-optimal path. Decision and Control. Proc. 42nd IEEE Conference on, v. 3, p. 2750-2755, 2003. ISSN 01912216.

[22] ISIDORI, A. Non linear control systems: an introduction. 2. ed. Berlin: SpringerVerlag, 1989. 479 p.

[23] NIJMEIJER, H. Nonlinear dynamical control systems. New York: Springer-Verlag, 1990. $467 \mathrm{p}$.

[24] ZABCZYK, J. Mathematical control theory: an introduction. Boston: Birkhäuser, 1992. $260 \mathrm{p}$. 